\title{
Predictions of the hidden-charm molecular states with the four quark components
}

\author{
Rui Chen ${ }^{1,2, a}$, Xiang Liu ${ }^{1,2, b}$, Yan-Rui Liu ${ }^{3,4, c}$, Shi-Lin Zhu ${ }^{5,6,7, d}$ \\ ${ }^{1}$ School of Physical Science and Technology, Lanzhou University, Lanzhou 730000, China \\ 2 Research Center for Hadron and CSR Physics, Lanzhou University and Institute of Modern Physics of CAS, Lanzhou 730000, China \\ ${ }^{3}$ School of Physics and Key Laboratory of Particle Physics and Particle Irradiation (MOE), Shandong University, Jinan 250100, China \\ ${ }^{4}$ Key Laboratory of Theoretical Physics, Institute of Theoretical Physics, CAS, Beijing 100190, China \\ ${ }^{5}$ School of Physics and State Key Laboratory of Nuclear Physics and Technology, Peking University, Beijing 100871, China \\ ${ }^{6}$ Collaborative Innovation Center of Quantum Matter, Beijing 100871, China \\ ${ }^{7}$ Center of High Energy Physics, Peking University, Beijing 100871, China
}

Received: 4 February 2016 / Accepted: 30 May 2016 / Published online: 10 June 2016

(c) The Author(s) 2016. This article is published with open access at Springerlink.com

\begin{abstract}
In this work, we study the $T \bar{T}$-type molecular systems systematically via the one pion exchange model, where $T$ denotes the narrow $J^{P}=1^{+} D_{1}$ meson or $2^{+} D_{2}^{*}$ meson and $\bar{T}$ is its antiparticle. With the effective potentials, we try to find the bound-state solutions of the corresponding systems, which provide crucial information of whether $T \bar{T}$ type molecular states exist. By our analysis, we predict some $T \bar{T}$-type molecular states which may be accessible at future experiments like $\mathrm{LHCb}$ and forthcoming BelleII.
\end{abstract}

\section{Introduction}

Searching for exotic states is a promising research topic full of opportunities and challenges in hadron physics. In 2013, the observation of the charged charmonium-like structure $Z_{c}(3900)$ from the BESIII [1] and Belle [2] Collaborations have inspired extensive discussions on the four-quark matter. Very recently, the LHCb Collaboration announced two hidden-charm pentaquarks, $P_{c}(4380)$ and $P_{c}(4450)$ [3], which have aroused theorists' interest in the five-quark matter again [4-8].

As one of the possible exotic hadron configurations, the multiquark state is a new hadronic matter type beyond the conventional $q \bar{q}$ meson and $q q q$ baryon. Among the multiquark states, the molecular state is a very popular configuration, which has been applied to understand some of the exper-

\footnotetext{
a e-mail: chenr2012@1zu.edu.cn

be-mail: xiangliu@1zu.edu.cn

c e-mail: yrliu@sdu.edu.cn

de-mail: zhus1@pku.edu.cn
}

imental observations of the near-threshold charmonium-like states, which are also named $X Y Z$ states.

In the past decade, the hidden-charm molecular states were studied extensively, since these investigations have a close relation to $X$ (3872) [9-15], $Y(3930)$ [15-17], $Y(4140)$ [1519], $Y$ (4274) [20-22], $Z_{b}(10610), Z_{b}(10650)[9,15,23,24]$, $Z^{+}(4430)[25,26]$, and the newly observed $P_{c}(4380)$ and $P_{c}$ (4450) [4-8]. As indicated in Refs. [27,28], the hiddencharm quantum number may be a crucial condition for the existence of the exotic molecular states. Thus, we can understand naturally why so many charmonium-like states (or bottomonium-like) states can be assigned to the hiddencharm molecular states.

With further experimental progress, more candidates of exotic states will be reported. For theorists, it is time to make reliable predictions of the hidden-charm molecular states. In this work, we focus on the hidden-charm molecular states with four quark components, which are composed of the charmed and anticharmed mesons. Their properties are determined by the corresponding components (charmed and anticharmed mesons).

In the heavy quark limit [29], the S-wave and P-wave charmed mesons can be grouped into three doublets, $H=$ $\left(0^{-}, 1^{-}\right), S=\left(0^{+}, 1^{+}\right)$, and $T=\left(1^{+}, 2^{+}\right)$. In Ref. [15], the hidden-charm molecular states composed of the charmed/anticharmed mesons in the $H$ doublet were studied, which are abbreviated as the $H \bar{H}$-type hidden-charm molecular states. Later, the possible $S \bar{S}$-type hidden-charm molecular states were predicted [30], which are constructed by the $P$-wave charm-strange mesons, $D_{s 0}(2317)$ and $D_{s 1}(2460)$, and their corresponding antiparticles, where $D_{s 0}(2317)$ and $D_{s 1}(2460)$ have very narrow widths. In addition, the authors of Ref. [31] analyzed the molecular systems composed of 
the charmed mesons in the $H$ doublet and $S$ doublet carefully, which are also named $H \bar{S}$-type hidden-charm molecular states.

Along this line, in this work, we continue to carry out the study of the $T \bar{T}$-type hidden-charm molecular states, which are composed of the charmed and anticharmed mesons in the $T$ doublet. The charmed mesons in the $T$ doublet have narrow widths. In Ref. [32], it is argued that the broad width of an open-charm meson probably results in unobservable bound states containing this meson. Thus, the charmed mesons in the $T$ doublet are suitable building blocks of the molecular states.

In order to investigate the $T \bar{T}$-type hidden-charm molecular states, we adopt the one pion exchange (OPE) model $[12,13,33]$ to deduce the effective potentials. With the obtained potentials and by solving Schrödinger equation, we finally get the bound-state solution and can judge whether the bound states exists. We need to specify that the S-D mixing effect $[23,24,34,35]$ and the coupled-channel effect [36,37] will be included in our calculation. The detailed deductions of the OPE potentials will be given in the next section. We hope that the present study may stimulate interest in searching for the $T \bar{T}$-type hidden-charm molecular states in experiments like $\mathrm{LHCb}$ and forthcoming BelleII.

A hadron-hadron bound state such as the deuteron is stable since its constituents do not decay. In the present case, both $D_{1}$ and $D_{2}^{*}$ have a width of tens of $\mathrm{MeV}$. Although a binding energy of several $\mathrm{MeV}$ for a possible molecule is smaller than the width of its constituent, some molecular type resonance may still result from the $T \bar{T}$ interaction. This character may be approximately studied by treating the mesons as stable states and encoding the width effects into some parameters. Such a point was discussed in Ref. [38] for the $N N^{*}(1440)$ interaction.

This paper is organized as follows. After the introduction, we present the deduction of the OPE potentials in Sect. 2. In Sect. 3, we present the corresponding numerical results. We summarize our results in Sect. 4.

\section{Deduction of the effective potentials}

In this section, we will derive the one-pion-exchange interaction potential between the charmed mesons in the $T$ doublet and their antiparticles. These charmed-anticharmed meson pairs can form three $S$-wave systems $D_{1} \bar{D}_{1}, D_{1} \bar{D}_{2}^{*}\left(D_{2}^{*} \bar{D}_{1}\right)$ and $D_{2}^{*} \bar{D}_{2}^{*}$, which are simply labeled $T \bar{T}$. The P-parity of the systems are all even, while the C-parity of the second system can be even or odd. In studying the bound-state problem, we need the masses of the charmed mesons in the $T$ doublet which are taken from the particle data group [39]: $M_{D_{1}}=2422.35 \mathrm{MeV}, M_{D_{2}}=2463.50 \mathrm{MeV}$.

\subsection{The wave functions}

To construct the wave function of a $T \bar{T}$ system with a given $\mathrm{C}$-parity, the convention for the C-parity transformation of a charmed meson should be addressed. It is required to be consistent with the convention in the Lagrangian (Eq. (8)). In addition, for the $D_{1} \bar{D}_{2}^{*}\left(D_{2}^{*} \bar{D}_{1}\right)$ system, we have a factor coming from the exchange of the two bosons appears. Along with the procedure in Ref. [40], we construct the flavor wave functions as follows. It is enough for us to consider only the neutral states because of the isospin invariance. For the $D_{1} \bar{D}_{1}$ and $D_{2}^{*} \bar{D}_{2}^{*}$ systems, one has

$\left|X_{T \bar{T}}^{0}[J]\right\rangle=\frac{1}{\sqrt{2}}\left(\left|T^{0} \bar{T}^{0}\right\rangle-x\left|T^{+} T^{-}\right\rangle\right)$,

where $x=1(-1)$ for the isovector (isoscalar) case and $J$ is the total angular momentum. For the $D_{1} \bar{D}_{2}^{*}\left(D_{2}^{*} \bar{D}_{1}\right)$ system, it is necessary to construct a G-parity eigenfunction in order to get the correct potential for a given C-parity [41]. With the convention $D_{1} \leftrightarrow \bar{D}_{1}, D_{2}^{*} \leftrightarrow-\bar{D}_{2}^{*}$ under the C-parity transformation, one finds

$$
\begin{aligned}
\left|X_{D_{1} \bar{D}_{2}^{*}}^{0}[J]\right\rangle= & \frac{1}{2 \sqrt{2}}\left(\left[\left|D_{1}^{0} \bar{D}_{2}^{* 0}\right\rangle+(-1)^{J-3}\left|\bar{D}_{2}^{* 0} D_{1}^{0}\right\rangle\right]\right. \\
& -x\left[\left|D_{1}^{+} D_{2}^{*-}\right\rangle+(-1)^{J-3}\left|D_{2}^{*-} D_{1}^{+}\right\rangle\right] \\
& -c\left[\left|\bar{D}_{1}^{0} D_{2}^{* 0}\right\rangle+(-1)^{J-3}\left|D_{2}^{* 0} \bar{D}_{1}^{0}\right\rangle\right] \\
& \left.+x c\left[\left|D_{1}^{-} D_{2}^{*+}\right\rangle+(-1)^{J-3}\left|D_{2}^{*+} D_{1}^{-}\right\rangle\right]\right),
\end{aligned}
$$

where $c=+1$ or -1 ; it is for the C-parity of the system. In the following discussions, we simply use the notation $D_{1} \bar{D}_{2}^{*}$ to denote the state $D_{1} \bar{D}_{2}^{*}+D_{2}^{*} \bar{D}_{1}$ or $D_{1} \bar{D}_{2}^{*}-D_{2}^{*} \bar{D}_{1}$.

The meson-antimeson molecular system is similar to the well-known deuteron [12,13] and the tensor force might be important. We also include the coupled-channel effects due to the $D$-wave interaction between the constituents. The allowed quantum numbers and possible coupled channels for the systems are

$$
\begin{aligned}
& D_{1} \bar{D}_{1}\left\{\begin{array}{l}
J^{P C}=0^{++}:\left|{ }^{1} S_{0}\right\rangle,\left|{ }^{5} D_{0}\right\rangle, \\
J^{P C}=1^{+-}:\left|{ }^{3} S_{1}\right\rangle,\left|{ }^{3} D_{1}\right\rangle, \\
J^{P C}=2^{++}:\left|{ }^{5} S_{2}\right\rangle,\left|{ }^{1} D_{2}\right\rangle,\left|{ }^{5} D_{2}\right\rangle,
\end{array}\right. \\
& D_{1} \bar{D}_{2}^{*}\left\{\begin{array}{l}
J^{P C}=1^{+ \pm}:\left|{ }^{3} S_{1}\right\rangle,\left|{ }^{3} D_{1}\right\rangle,\left|{ }^{5} D_{1}\right\rangle,\left|{ }^{7} D_{1}\right\rangle, \\
J^{P C}=2^{+ \pm}:\left|{ }^{5} S_{2}\right\rangle,\left|{ }^{3} D_{2}\right\rangle,\left|{ }^{5} D_{2}\right\rangle,\left|{ }^{7} D_{2}\right\rangle, \\
J^{P C}=3^{+ \pm}:\left|{ }^{7} S_{3}\right\rangle,\left|{ }^{3} D_{3}\right\rangle,\left|{ }^{5} D_{3}\right\rangle,\left|{ }^{7} D_{3}\right\rangle,
\end{array}\right. \\
& D_{2}^{*} \bar{D}_{2}^{*}\left\{\begin{array}{l}
J^{P C}=0^{++}:\left|{ }^{1} S_{0}\right\rangle,\left|{ }^{5} D_{0}\right\rangle, \\
J^{P C}=1^{+-}:\left|{ }^{3} S_{1}\right\rangle,\left|{ }^{3} D_{1}\right\rangle,\left|{ }^{7} D_{1}\right\rangle, \\
J^{P C}=2^{++}:\left|{ }^{5} S_{2}\right\rangle,\left|{ }^{1} D_{2}\right\rangle,\left|{ }^{5} D_{2}\right\rangle,\left|{ }^{9} D_{2}\right\rangle, \\
J^{P C}=4^{++}:\left|{ }^{9} S_{4}\right\rangle,\left|{ }^{5} D_{4}\right\rangle,\left|{ }^{9} D_{4}\right\rangle,
\end{array}\right.
\end{aligned}
$$

where the notation $\left.\left.\right|^{2 S+1} L_{J}\right\rangle$ is used. Contributions from the higher partial wave channels are significantly suppressed and 
we do not include such channels here. The P-parity is always +1 . The C-parity for the $D_{1} \bar{D}_{1}$ and $D_{2}^{*} \bar{D}_{2}^{*}$ systems is determined with $C=(-1)^{L+S}$ while the C-parity for the $D_{1} \bar{D}_{2}^{*}$ system is not constrained by symmetry. Since the orbital angular momentum $L=0,2$, one observes that the total spin $S$ of the system is always odd (even) for the $D_{1} \bar{D}_{1}$ and $D_{2}^{*} \bar{D}_{2}^{*}$ states with negative (positive) C-parity while both odd and even $S$ are allowed for the $D_{1} \bar{D}_{2}^{*}$ states.

The molecular system composed of two mesons $A B$ is defined as

$$
\begin{aligned}
\left|A B\left({ }^{2 S+1} L_{J}\right)\right\rangle & =\sum_{m_{A}, m_{B}, m_{S}, m_{L}} C_{s_{A}, m_{A} ; s_{B}, m_{B}}^{S, m_{S}} C_{S, m_{S} ; L, m_{L}}^{J, M} \epsilon^{m_{A}} \epsilon^{m_{B}}\left|Y_{L, m_{L}}\right\rangle .
\end{aligned}
$$

Here, $C_{s_{A}, m_{A} ; s_{B}, m_{B}}^{S, m_{S}}$ and $C_{S, m_{S} ; L, m_{L}}^{J, M}$ denote the CG coefficients, $\left|Y_{L, m_{L}}\right\rangle$ is the spherical harmonic function, and $\epsilon^{m}$ is the polarization vector $\varepsilon^{m}$ for the axial-vector meson or the polarization tensor $\xi^{m}$ for the tensor meson. In the static limit, the special expressions for the polarization vector are $\varepsilon^{ \pm 1}=\frac{1}{\sqrt{2}}(0, \pm 1, i, 0)$ and $\varepsilon^{0}=(0,0,0,-1)$. The polarization tensor $\xi^{m}$ can be constructed from the polarization

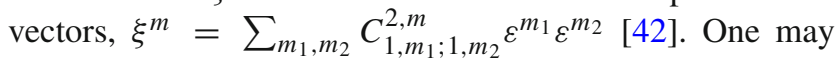
use each z-component $(M=0, \pm 1, \ldots, \pm J)$ to derive the potential.

\subsection{The effective Lagrangian}

We will study the meson-antimeson interaction in a onepion-exchange (OPE) model. The effective potential is derived using the effective Lagrangian with the heavy quark symmetry and chiral symmetry, which can be written in a compact form [43],

$\mathcal{L}_{\mathbb{P}}=i k\left\langle T_{b}^{(Q) \mu} \mid A_{b a} \gamma_{5} \bar{T}_{a \mu}^{(Q)}\right\rangle+i k\left\langle\bar{T}_{a}^{(\bar{Q}) \mu} \mid A_{a b} \gamma_{5} T_{b \mu}^{(\bar{Q})}\right\rangle$.

Here, the axial field $A_{\mu}$ is constructed with the pion field

$$
\begin{aligned}
A_{\mu} & =\frac{1}{2}\left(\zeta^{\dagger} \partial_{\mu} \zeta-\zeta \partial_{\mu} \zeta^{\dagger}\right), \\
\zeta & =\exp \left(i \mathbb{P} / f_{\pi}\right), \\
\mathbb{P} & =\left(\begin{array}{cc}
\frac{\pi^{0}}{\sqrt{2}} & \pi^{+} \\
\pi^{-} & -\frac{\pi^{0}}{\sqrt{2}}
\end{array}\right),
\end{aligned}
$$

where $f_{\pi}=132 \mathrm{MeV}$ is the pion decay constant. The $T$ doublet now is expressed in a form of the superfield

$$
\begin{aligned}
T_{a}^{(Q) \mu}= & \frac{1+w}{2}\left[P_{2 a}^{*(Q) \mu \nu} \gamma_{\nu}-\sqrt{\frac{3}{2}} P_{1 a v}^{(Q)} \gamma_{5}\right. \\
& \left.\left(g^{\mu \nu}-\frac{1}{3} \gamma^{\nu}\left(\gamma^{\mu}-v^{\mu}\right)\right)\right], \\
P_{2 a}^{*(Q)}= & \left(D_{2}^{* 0}, D_{2}^{*+}\right), \quad P_{1}^{(Q)}=\left(D_{1}^{0}, D_{1}^{+}\right),
\end{aligned}
$$

where the four-velocity $v=(1, \overrightarrow{0})$ in the static approximation. The normalization for the mesons involved are $\left\langle 0\left|P_{1}^{\mu}\right| Q \bar{q}\left(1^{+}\right)\right\rangle=\varepsilon^{\mu} \sqrt{M_{P_{1}}}$ and $\left\langle 0\left|P_{2}^{* \mu \nu}\right| Q \bar{q}\left(2^{+}\right)\right\rangle=$ $\xi^{\mu \nu} \sqrt{M_{P_{2}^{*}}}$. The antimeson doublet containing a heavy antiquark $\bar{Q}$ is obtained from the charge conjugate operation,

$$
\begin{aligned}
T_{a}^{(\bar{Q}) \mu}= & C\left(\mathcal{C} T_{a}^{(Q) \mu} \mathcal{C}^{-1}\right)^{T} C^{-1} \\
= & {\left[P_{2 a}^{*(\bar{Q}) \mu \nu} \gamma_{\nu}-\sqrt{\frac{3}{2}} P_{1 a \nu}^{(\bar{Q})} \gamma_{5}\left(g^{\mu \nu}\right.\right.} \\
& \left.\left.-\frac{1}{3} \gamma^{\nu}\left(\gamma^{\mu}-v^{\mu}\right) \gamma^{\nu}\right)\right] \frac{1-t}{2},
\end{aligned}
$$

where the transpose is for the $\gamma$ matrices, $\mathcal{C}$ is the charge conjugate operator and the matrix $C=i \gamma^{2} \gamma^{0}$. The convention for the $\mathrm{C}$-parity transformation for meson and antimeson fields is

$P_{2 a}^{*(\bar{Q}) \mu \nu}=-\mathcal{C} P_{2 a}^{*(Q) \mu \nu} \mathcal{C}^{-1}, \quad P_{1 a \nu}^{(\bar{Q})}=\mathcal{C} P_{1 a \nu}^{(Q)} \mathcal{C}^{-1}$,

and the hermitian conjugate fields are defined by

$\bar{T}_{a}^{(Q) \mu}=\gamma_{0} T_{a}^{(Q) \mu \dagger} \gamma_{0}, \quad \bar{T}_{a \mu}^{(\bar{Q})}=\gamma_{0} T_{a \mu}^{(\bar{Q}) \dagger} \gamma_{0}$.

In the compact Lagrangian, $\langle\ldots\rangle$ denotes the trace in the spin and flavor space.

Expanding Eq. (7), one obtains the interaction terms of the $D_{1}\left(D_{2}^{*}\right)$ and $\bar{D}_{1}\left(\bar{D}_{2}^{*}\right)$ mesons with the pion,

$$
\begin{aligned}
\mathcal{L}_{D_{1} D_{1} \mathbb{P}}= & i \frac{5 k}{3 f_{\pi}} v^{\mu} \varepsilon_{\mu \nu \alpha \beta} D_{1 b}^{\nu} D_{1 a}^{\beta \dagger} \partial^{\alpha} \mathbb{P}_{b a} \\
& -i \frac{5 k}{3 f_{\pi}} v^{\mu} \varepsilon_{\mu \nu \alpha \beta} \bar{D}_{1 a}^{\nu \dagger} \bar{D}_{1 b}^{\beta} \partial^{\alpha} \mathbb{P}_{a b}, \\
\mathcal{L}_{D_{2}^{*} D_{2}^{*} \mathbb{P}}= & -2 i \frac{k}{f_{\pi}} v^{\lambda} \varepsilon_{\lambda \nu \alpha \sigma} D_{2 b}^{* \mu \nu} D_{2 a \mu}^{* \sigma \dagger} \partial^{\alpha} \mathbb{P}_{b a} \\
& -2 i \frac{k}{f_{\pi}} v^{\lambda} \varepsilon_{\lambda \nu \alpha \sigma} \bar{D}_{2 a \mu}^{* \sigma \dagger} \bar{D}_{2 b}^{* \mu \nu} \partial^{\alpha} \mathbb{P}_{a b}, \\
\mathcal{L}_{D_{1} D_{2}^{*} \mathbb{P}}= & -\sqrt{\frac{2}{3}} \frac{k}{f_{\pi}}\left(D_{2 b}^{* \mu \nu} D_{1 a \mu}^{\dagger}+D_{1 b \mu} D_{2 a}^{* \mu \nu \dagger}\right) \partial_{v} \mathbb{P}_{b a} \\
& +\sqrt{\frac{2}{3}} \frac{k}{f_{\pi}}\left(\bar{D}_{2 a}^{* \mu \nu \dagger} \bar{D}_{1 b \mu}+\bar{D}_{1 a \mu}^{\dagger} \bar{D}_{2 b}^{* \mu \nu}\right) \partial_{\nu} \mathbb{P}_{a b} .
\end{aligned}
$$

The lowest charmed meson doublet is $H=\left(0^{-}, 1^{-}\right)$. The coupling constant $g$ for the $H-H-\pi$ interaction can be extracted from the strong decay $D^{*} \rightarrow D \pi: g=0.59 \pm$ $0.07 \pm 0.01$ [44]. In this work, the coupling constant $k$ is taken to have the same value as $g[26,37,45]$.

\subsection{The OPE effective potential}

With the above effective Lagrangians, we can write down the amplitudes for the t-channel scattering processes, which are related to the required potentials. The involved processes 
are $D_{1} \bar{D}_{1} \rightarrow D_{1} \bar{D}_{1}, D_{1} \bar{D}_{2}^{*} \rightarrow D_{1} \bar{D}_{2}^{*}, D_{1} \bar{D}_{2}^{*} \rightarrow D_{2}^{*} \bar{D}_{1}$, $D_{2}^{*} \bar{D}_{1} \rightarrow D_{1} \bar{D}_{2}^{*}, D_{2}^{*} \bar{D}_{1} \rightarrow D_{2}^{*} \bar{D}_{1}$, and $D_{2}^{*} \bar{D}_{2}^{*} \rightarrow D_{2}^{*} \bar{D}_{2}^{*}$. Generally, the effective potential in the momentum space is related to the obtained scattering amplitude through the Breit approximation. Here, we take the process $D_{1} \bar{D}_{1} \rightarrow D_{1} \bar{D}_{1}$ as an example. The relation between the effective potential $\mathcal{V}$ and scattering amplitude $\mathcal{M}$ is

$\mathcal{V}\left[D_{1} \bar{D}_{1} \rightarrow D_{1} \bar{D}_{1}\right](\vec{q})=-\frac{\mathcal{M}\left(D_{1} \bar{D}_{1} \rightarrow D_{1} \bar{D}_{1}\right)}{\sqrt{\prod_{i} 2 M_{i} \prod_{f} 2 M_{f}}}$,

where $M_{i}$ and $M_{f}$ denote the masses of the initial and final states, respectively. We calculate the scattering amplitude $\mathcal{M}\left(D_{1} \bar{D}_{1} \rightarrow D_{1} \bar{D}_{1}\right)$ in the one-pion-exchange approximation. In addition, one obtains the effective potential in the coordinate space by performing the Fourier transformation,

$$
\begin{aligned}
\mathcal{V} & {\left[D_{1} \bar{D}_{1} \rightarrow D_{1} \bar{D}_{1}\right](\vec{r}) } \\
& =\int \frac{d^{3} \vec{q}}{(2 \pi)^{3}} e^{i \vec{q} \cdot(r)} \mathcal{F}^{2}\left(q^{2}, m_{E}^{2}\right) \mathcal{V}\left[D_{1} \bar{D}_{1} \rightarrow D_{1} \bar{D}_{1}\right](\vec{q}),
\end{aligned}
$$

where $\mathcal{F}\left(q^{2}, m_{E}^{2}\right)=\left(\Lambda^{2}-m_{E}^{2}\right) /\left(\Lambda^{2}-q^{2}\right)$ is the monopole type form factor with $m_{E}$ the mass of the exchanged meson. The monopole form factor is introduced at each interaction vertex to compensate the off shell effect of the exchanged meson and describe the structure effect of the vertex. In practice, the phenomenological cutoff $\Lambda$ is around one to several $\mathrm{GeV}$.

Following the above procedure, we obtain the OPE potentials for the $D_{1} \bar{D}_{1}, D_{1} \bar{D}_{2}^{*}$, and $D_{2}^{*} \bar{D}_{2}^{*}$ systems. The form of the final potentials for these systems may be summarized into a compact form,

$$
\begin{aligned}
\mathcal{V}_{D_{1} \bar{D}_{1}}(I, J, r)= & \mathcal{G}(I) \times \mathcal{V}_{1}, \\
\mathcal{V}_{D_{1} \bar{D}_{2}^{*}}(I, J, r)= & \frac{1}{2} \mathcal{G}(I) \\
& \times\left(\mathcal{V}_{5}+\mathcal{V}_{8}-c(-1)^{J-3}\left(\mathcal{V}_{6}+\mathcal{V}_{11}\right)\right),
\end{aligned}
$$

$\mathcal{V}_{D_{2}^{*} \bar{D}_{2}^{*}}(I, J, r)=\mathcal{G}(I) \times \mathcal{V}_{10}$.

Here, $I$ is the isospin of the state and $\mathcal{G}(I)$ is defined as an isospin factor, with the value $\mathcal{G}(0)=3 / 2$ and $\mathcal{G}(1)=-1 / 2$. One gets these potentials by sandwiching the above $(I, J)$ independent potentials between the states in Eqs. (1) and (2).

We take the $D_{1} \bar{D}_{1}$ system as an example to illustrate the structure of the potentials. Now

$$
\begin{aligned}
\mathcal{V}_{D_{1} \bar{D}_{1}}(I, J, r)= & \left\langle X_{D_{1} \bar{D}_{1}}^{0}[J]\right| \mathcal{V} \\
& \times\left[D_{1} \bar{D}_{1} \rightarrow D_{1} \bar{D}_{1}\right](\vec{r})\left|X_{D_{1} \bar{D}_{1}}^{0}[J]\right\rangle \\
\equiv & \left\langle X_{D_{1} \bar{D}_{1}}^{0}[J]\left|\hat{\mathcal{V}}_{1}\right| X_{D_{1} \bar{D}_{1}}^{0}[J]\right\rangle \\
= & \mathcal{G}(I) \times \mathcal{V}_{1},
\end{aligned}
$$

where $\hat{\mathcal{V}}_{1}$ has the form $\hat{\mathcal{V}}_{1}=$ const $\times\left[\mathcal{E}_{1} f(r)+\mathcal{S}_{1} g(r)\right]$. Here, $\mathcal{E}_{1}\left(\mathcal{S}_{1}\right)$ is the spin-spin (tensor) operator and $f(r)(g(r))$ is a potential function depending on the cutoff $\Lambda$, the pion mass $m_{\pi}$, and the radial coordinate $r$. Obviously, $\mathcal{V}_{1}$ is a $n \times n$ matrix, where $n$ is the number of the coupled channels in Eq. (3), which depends on the total angular momentum $J$. The explicit matrix elements for the operator $\mathcal{E}_{1}$ are

$$
\begin{aligned}
& \left\langle\mathcal{E}_{1}[J=0]\right\rangle=\left(\begin{array}{cc}
\left\langle{ }^{1} S_{0}\left|\mathcal{E}_{1}\right|^{1} S_{0}\right\rangle & \left\langle{ }^{1} S_{0}\left|\mathcal{E}_{1}\right|^{5} D_{0}\right\rangle \\
\left.{ }^{5} D_{0}\left|\mathcal{E}_{1}\right|^{1} S_{0}\right\rangle & \left\langle{ }^{5} D_{0}\left|\mathcal{E}_{1}\right|^{5} D_{0}\right\rangle
\end{array}\right)=\left(\begin{array}{cc}
2 & 0 \\
0 & -1
\end{array}\right),
\end{aligned}
$$

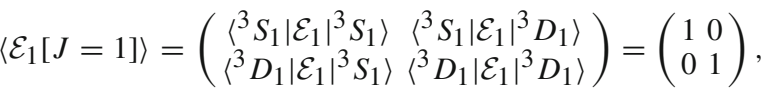

$$
\begin{aligned}
& \left\langle\mathcal{E}_{1}[J=2]\right\rangle=\left(\begin{array}{lll}
\left\langle{ }^{5} S_{2}\left|\mathcal{E}_{1}\right|^{5} S_{2}\right\rangle & \left\langle{ }^{5} S_{2}\left|\mathcal{E}_{1}\right|{ }^{1} D_{2}\right\rangle & \left\langle{ }^{5} S_{2}\left|\mathcal{E}_{1}\right|^{5} D_{2}\right\rangle \\
\left\langle{ }^{1} D_{2} \mid \mathcal{E}_{1}{ }^{5} S_{2}\right\rangle & \left\langle{ }^{1} D_{2}\left|\mathcal{E}_{1}\right|{ }^{1} D_{2}\right\rangle & \left\langle{ }^{1} D_{2}\left|\mathcal{E}_{1}\right|{ }^{5} D_{2}\right\rangle \\
\left.{ }^{5} D_{2}\left|\mathcal{E}_{1}\right|^{5} S_{2}\right\rangle & \left\langle{ }^{5} D_{2}\left|\mathcal{E}_{1}\right|{ }^{1} D_{2}\right\rangle & \left\langle{ }^{5} D_{2}\left|\mathcal{E}_{1}\right|{ }^{5} D_{2}\right\rangle
\end{array}\right) \\
& =\left(\begin{array}{ccc}
-1 & 0 & 0 \\
0 & 2 & 0 \\
0 & 0 & -1
\end{array}\right) \text {, }
\end{aligned}
$$

and those for $\mathcal{S}_{1}$ are

$$
\begin{aligned}
& \left\langle\mathcal{S}_{1}[J=0]\right\rangle=\left(\begin{array}{cc}
0 & \sqrt{2} \\
\sqrt{2} & 2
\end{array}\right)_{J=0}, \\
& \left\langle\mathcal{S}_{1}[J=1]\right\rangle=\left(\begin{array}{cc}
0 & -\sqrt{2} \\
-\sqrt{2} & 1
\end{array}\right)_{J=1}, \\
& \left\langle\mathcal{S}_{1}[J=2]\right\rangle=\left(\begin{array}{ccc}
0 & \sqrt{\frac{2}{5}} & -\sqrt{\frac{14}{5}} \\
\sqrt{\frac{2}{5}} & 0 & -\frac{2}{\sqrt{7}} \\
-\sqrt{\frac{14}{5}}-\frac{2}{\sqrt{7}} & -\frac{3}{7}
\end{array}\right)_{J=2} .
\end{aligned}
$$

When the quantum numbers are the same, the transitions between the states $D_{1} \bar{D}_{1}, D_{1} \bar{D}_{2}^{*}$, and $D_{2}^{*} \bar{D}_{2}^{*}$ may happen. We also consider such coupled-channel effects in this paper. The involved transitions include $D_{1} \bar{D}_{1} \leftrightarrow D_{1} \bar{D}_{2}^{*}, D_{1} \bar{D}_{1} \leftrightarrow$ $D_{2}^{*} \bar{D}_{1}, D_{1} \bar{D}_{1} \leftrightarrow D_{2}^{*} \bar{D}_{2}^{*}, D_{1} \bar{D}_{2}^{*} \leftrightarrow D_{2}^{*} \bar{D}_{2}^{*}$, and $D_{2}^{*} \bar{D}_{1} \leftrightarrow$ $D_{2}^{*} \bar{D}_{2}^{*}$. Relevant transition potentials are $\hat{\mathcal{V}}_{2,3,4,7,9}$. We will show the form of the final potentials in the next section. We collect all the defined subpotentials $\hat{\mathcal{V}}_{k}(k=1,2, \ldots, 11)$ in Eqs. (A3)-(13) in Appendix A. The related matrix elements are given in Tables 7 and 8 .

\section{Numerical results}

With the effective potentials (12)-(14), we can search for the bound-state solutions by solving the coupled-channel Schrödinger equation,

$\frac{1}{2 \mu}\left(-\nabla^{2}+\frac{l(l+1)}{r^{2}}\right) \psi(r)+(V(r)-E) \psi(r)=0$,

where $\nabla^{2}=\frac{1}{r^{2}} \frac{\mathrm{d}}{\mathrm{d} r} r^{2} \frac{\mathrm{d}}{\mathrm{d} r}, V(r)=\mathcal{V}_{D_{1} \bar{D}_{1}}(I, J, r), \mathcal{V}_{D_{1} \bar{D}_{2}^{*}}$ $(I, J, r)$, or $\mathcal{V}_{D_{2}^{*} \bar{D}_{2}^{*}}(I, J, r)$, and $\mu$ is the reduced mass of the 
Table 1 The bound-state solutions (the binding energy $E$ and the root-mean-square radius $r_{\text {RMS }}$ ) for the pure $D_{1} \bar{D}_{1}$ and $D_{2}^{*} \bar{D}_{2}^{*}$ systems
$E, r_{\mathrm{RMS}}$, and $\Lambda$ are in units of $\mathrm{MeV}, \mathrm{fm}$, and $\mathrm{GeV}$, respectively. The notation $\cdots$ means that no binding solution is found

\begin{tabular}{|c|c|c|c|c|c|c|c|c|}
\hline Systems & $I^{G}\left(J^{P C}\right)$ & $\Lambda$ & $E$ & $r_{\text {RMS }}$ & $I^{G}\left(J^{P C}\right)$ & $\Lambda$ & $E$ & $r_{\mathrm{RMS}}$ \\
\hline \multirow[t]{9}{*}{$D_{1} \bar{D}_{1}$} & \multirow[t]{3}{*}{$0^{+}\left(0^{++}\right)$} & 1.22 & -0.42 & 4.21 & \multirow[t]{3}{*}{$1^{-}\left(0^{++}\right)$} & $\ldots$ & $\ldots$ & $\ldots$ \\
\hline & & 1.32 & -3.41 & 1.67 & & $\ldots$ & $\ldots$ & $\ldots$ \\
\hline & & 1.42 & -9.64 & 1.06 & & $\ldots$ & $\ldots$ & $\ldots$ \\
\hline & \multirow[t]{3}{*}{$0^{-}\left(1^{+-}\right)$} & 1.96 & -1.34 & 2.59 & \multirow[t]{3}{*}{$1^{+}\left(1^{+-}\right)$} & $\ldots$ & $\ldots$ & $\ldots$ \\
\hline & & 2.06 & -4.35 & 1.50 & & $\ldots$ & $\ldots$ & $\ldots$ \\
\hline & & 2.16 & -9.38 & 1.06 & & $\ldots$ & $\ldots$ & $\ldots$ \\
\hline & \multirow[t]{3}{*}{$0^{+}\left(2^{++}\right)$} & 3.49 & -1.02 & 3.14 & \multirow[t]{3}{*}{$1^{-}\left(2^{++}\right)$} & 4.75 & -1.43 & 2.44 \\
\hline & & 3.59 & -2.27 & 2.20 & & 4.85 & -3.68 & 1.55 \\
\hline & & 3.69 & -4.12 & 1.70 & & 4.95 & -7.08 & 1.14 \\
\hline \multirow[t]{15}{*}{$D_{2}^{*} \bar{D}_{2}^{*}$} & \multirow[t]{3}{*}{$0^{+}\left(0^{++}\right)$} & 1.10 & -0.33 & 4.44 & \multirow[t]{3}{*}{$1^{-}\left(0^{++}\right)$} & $\ldots$ & $\ldots$ & $\ldots$ \\
\hline & & 1.20 & -3.21 & 1.74 & & $\ldots$ & $\ldots$ & $\ldots$ \\
\hline & & 1.30 & -9.46 & 1.08 & & $\ldots$ & $\ldots$ & $\ldots$ \\
\hline & \multirow[t]{3}{*}{$0^{-}\left(1^{+-}\right)$} & 1.22 & -0.07 & 5.76 & \multirow[t]{3}{*}{$1^{+}\left(1^{+-}\right)$} & $\ldots$ & $\ldots$ & $\ldots$ \\
\hline & & 1.32 & -2.04 & 2.12 & & $\ldots$ & $\ldots$ & $\ldots$ \\
\hline & & 1.42 & -6.74 & 1.25 & & $\ldots$ & $\ldots$ & $\ldots$ \\
\hline & \multirow[t]{3}{*}{$0^{+}\left(2^{++}\right)$} & 1.74 & -0.42 & 4.16 & \multirow[t]{3}{*}{$1^{-}\left(2^{++}\right)$} & $\ldots$ & $\ldots$ & $\ldots$ \\
\hline & & 1.84 & -2.56 & 1.91 & & $\ldots$ & $\ldots$ & $\ldots$ \\
\hline & & 1.94 & -6.80 & 1.23 & & $\ldots$ & $\ldots$ & $\ldots$ \\
\hline & \multirow[t]{3}{*}{$0^{-}\left(3^{+-}\right)$} & 2.70 & -0.24 & 4.97 & \multirow[t]{3}{*}{$1^{+}\left(3^{+-}\right)$} & $\ldots$ & $\ldots$ & $\ldots$ \\
\hline & & 2.90 & -3.08 & 1.85 & & $\ldots$ & $\ldots$ & $\ldots$ \\
\hline & & 3.10 & -9.94 & 1.11 & & $\ldots$ & $\ldots$ & $\ldots$ \\
\hline & \multirow[t]{3}{*}{$0^{+}\left(4^{++}\right)$} & 3.90 & -0.29 & 4.86 & \multirow[t]{3}{*}{$1^{-}\left(4^{++}\right)$} & 3.58 & -0.01 & 6.15 \\
\hline & & 4.20 & -2.61 & 2.07 & & 3.78 & -3.26 & 1.64 \\
\hline & & 4.50 & -8.23 & 1.26 & & 3.98 & -12.09 & 0.89 \\
\hline
\end{tabular}

system. The wave function $\psi(r)$ is a column matrix depending on $V(r)$.

Then we adopt the FESSDE program $[46,47]$ to calculate the energy eigenvalue $E$ in units of $\mathrm{MeV}$. If a bound-state solution exists, the binding energy $E$ is defined as the mass difference $M_{\text {system }}-M_{\text {constituents }}$. We further calculate the corresponding root-mean-square (RMS) radius $r_{\mathrm{RMS}}$ in units of fm. $E$ and $r_{\mathrm{RMS}}$ depend on the cutoff parameter $\Lambda$, which varies from 0.5 to $5 \mathrm{GeV}$.

\subsection{The pure $D_{1} \bar{D}_{1}, D_{1} \bar{D}_{2}^{*}$, and $D_{2}^{*} \bar{D}_{2}^{*}$ systems}

In this subsection, we present three sets of numerical results for the pure $D_{1} \bar{D}_{1}, D_{1} \bar{D}_{2}^{*}$, and $D_{2}^{*} \bar{D}_{2}^{*}$ systems in Tables 1 and 2 . There are 14 isoscalar and four isovector bound-state solutions in the $T \bar{T}$ systems. The $\Lambda$ dependence of the obtained binding energy is shown in Fig. 1. The results are very sensitive to the cutoff parameter, which is a common feature of these types of investigations $[9,15,23,24,26,30,31,34$ $37,40,43,48-51]$. At present, we cannot determine its value for the present systems from experimental observables. One should be cautious that the binding solutions for the mesonantimeson systems are possible only when the cutoff falls into a reasonable range. A repulsive potential or attractive potential with an inappropriate cutoff does not result in bound states.

If we compare all the bound-state solutions system by system, we notice an inequality $\Lambda_{J=0}^{I=0}\left(D_{1} \bar{D}_{1}\right)<\Lambda_{J=1}^{I=0}\left(D_{1} \bar{D}_{1}\right)$ $<\Lambda_{J=2}^{I=0}\left(D_{1} \bar{D}_{1}\right)$ if the binding energies are fixed at roughly the same value. To some extent, the existence possibility of one molecule is related to the value of the cutoff $\Lambda$. We notice $P\left(0^{+}\left(0^{++}\right)\right)>P\left(0^{-}\left(1^{+-}\right)\right)>P\left(0^{+}\left(2^{++}\right)\right)$, where $P\left(0^{+}\left(0^{++}\right)\right)$denotes the existence possibility for the $D_{1} \bar{D}_{1}$ with $I^{G}\left(J^{P C}\right)=0^{+}\left(0^{++}\right)$. In other words, the $D_{1} \bar{D}_{1}$ with $I^{G}\left(J^{P C}\right)=0^{+}\left(0^{++}\right)$is the most stable loosely bound structure, followed by $0^{-}\left(1^{+-}\right)$and $0^{+}\left(2^{++}\right)$. This binding feature results certainly from the potentials. Since the main contribution comes from the S-wave interaction, the matrix elements for the operator $\mathcal{E}_{1}$ in the diagonal S-wave channels, $\left\langle{ }^{1} S_{0}\left|\mathcal{E}_{1}\right|{ }^{1} S_{0}\right\rangle=2,\left\langle{ }^{3} S_{1}\left|\mathcal{E}_{1}\right|{ }^{3} S_{1}\right\rangle=1$, and $\left\langle{ }^{5} S_{2}\left|\mathcal{E}_{1}\right|{ }^{5} S_{2}\right\rangle=-1$ affect dominantly the attraction strength. Therefore, the $0^{++}$ state is the one most easily bound. The isoscalar scalar $D_{1} \bar{D}_{1}$ system may be easiest to detect in future experiments. Moreover, we also notice the same trend in the isoscalar $D_{1} \bar{D}_{2}^{*}$ and isoscalar $D_{2}^{*} \bar{D}_{2}^{*}$ cases.

In terms of the isovector states, there are only four binding solutions, which are the $D_{1} \bar{D}_{1}$ system with $1^{-}\left(2^{++}\right), D_{1} \bar{D}_{2}^{*}$ 
Table 2 The bound-state solutions (the binding energy $E$ and the root-mean-square radius $r_{\mathrm{RMS}}$ ) for the pure $D_{1} \bar{D}_{2}^{*}$ system

$E, r_{\mathrm{RMS}}$, and $\Lambda$ are in units of $\mathrm{MeV}$, fm, and $\mathrm{GeV}$, respectively. The notation $\cdots$ means that no binding solution is found

\begin{tabular}{|c|c|c|c|c|c|c|c|c|}
\hline Systems & $I^{G}\left(J^{P C}\right)$ & $\Lambda$ & $E$ & $r_{\mathrm{RMS}}$ & $I^{G}\left(J^{P C}\right)$ & $\Lambda$ & $E$ & $r_{\mathrm{RMS}}$ \\
\hline \multirow[t]{18}{*}{$D_{1} \bar{D}_{2}^{*}$} & \multirow[t]{3}{*}{$0^{-}\left(1^{+-}\right)$} & 1.32 & -0.17 & 5.11 & \multirow[t]{3}{*}{$1^{+}\left(1^{+-}\right)$} & $\ldots$ & $\ldots$ & $\ldots$ \\
\hline & & 1.42 & -2.62 & 1.84 & & $\ldots$ & $\ldots$ & $\ldots$ \\
\hline & & 1.52 & -8.13 & 1.11 & & $\ldots$ & $\ldots$ & $\ldots$ \\
\hline & \multirow[t]{3}{*}{$0^{-}\left(2^{+-}\right)$} & 3.36 & -0.53 & 3.75 & \multirow[t]{3}{*}{$1^{+}\left(2^{+-}\right)$} & $\ldots$ & $\ldots$ & $\ldots$ \\
\hline & & 3.46 & -2.25 & 1.97 & & $\ldots$ & $\ldots$ & $\ldots$ \\
\hline & & 3.56 & -5.25 & 1.32 & & $\ldots$ & $\ldots$ & $\ldots$ \\
\hline & \multirow[t]{3}{*}{$0^{-}\left(3^{+-}\right)$} & 3.03 & -1.17 & 2.96 & \multirow[t]{3}{*}{$1^{+}\left(3^{+-}\right)$} & 4.71 & -0.82 & 3.14 \\
\hline & & 3.13 & -2.70 & 2.06 & & 4.81 & -2.56 & 1.84 \\
\hline & & 3.23 & -5.04 & 1.57 & & 4.91 & -5.35 & 1.30 \\
\hline & \multirow[t]{3}{*}{$0^{+}\left(1^{++}\right)$} & 1.34 & -0.31 & 4.48 & \multirow[t]{3}{*}{$1^{-}\left(1^{++}\right)$} & $\ldots$ & $\ldots$ & $\ldots$ \\
\hline & & 1.44 & -2.94 & 1.77 & & $\ldots$ & $\ldots$ & $\ldots$ \\
\hline & & 1.54 & -8.50 & 1.10 & & $\ldots$ & $\ldots$ & $\ldots$ \\
\hline & \multirow[t]{3}{*}{$0^{+}\left(2^{++}\right)$} & 2.30 & -0.37 & 4.29 & \multirow[t]{3}{*}{$1^{-}\left(2^{++}\right)$} & $\ldots$ & $\ldots$ & $\ldots$ \\
\hline & & 2.40 & -2.08 & 2.08 & & $\ldots$ & $\ldots$ & $\ldots$ \\
\hline & & 2.50 & -5.33 & 1.36 & & $\ldots$ & $\ldots$ & $\ldots$ \\
\hline & \multirow[t]{3}{*}{$0^{+}\left(3^{++}\right)$} & 3.91 & -0.25 & 5.06 & \multirow[t]{3}{*}{$1^{-}\left(3^{++}\right)$} & 3.98 & -1.04 & 2.79 \\
\hline & & 4.21 & -3.48 & 1.82 & & 4.08 & -3.35 & 1.60 \\
\hline & & 4.45 & -9.89 & 1.16 & & 4.18 & -7.08 & 1.12 \\
\hline
\end{tabular}
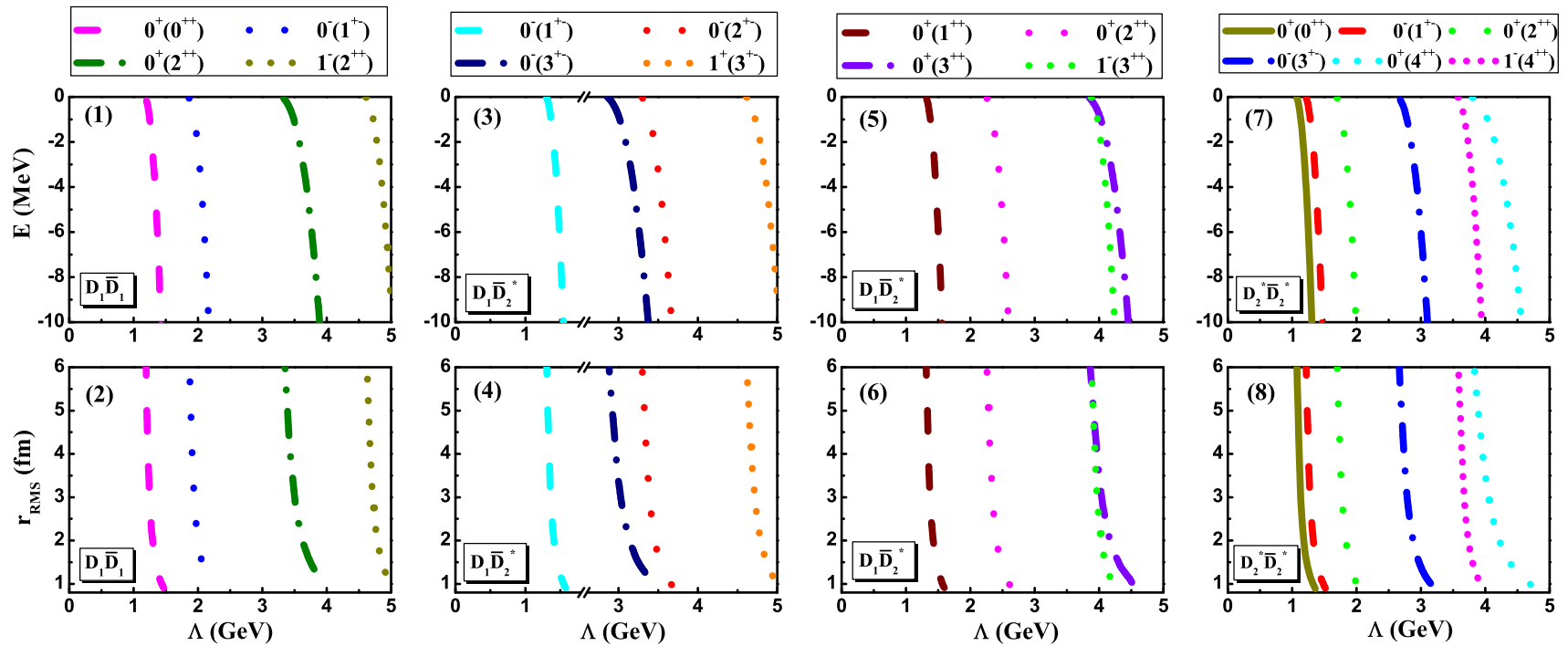

Fig. 1 The $\Lambda$ dependence of the bound-state solutions (the binding energy $E$ and the root-mean-square radius $r_{\mathrm{RMS}}$ ) for the pure $D_{1} \bar{D}_{1}, D_{1} \bar{D}_{2}^{*}$, and $D_{2}^{*} \bar{D}_{2}^{*}$ systems with all the possible configurations

with $1^{+}\left(3^{+-}\right), 1^{-}\left(3^{++}\right)$and $D_{2}^{*} \bar{D}_{2}^{*}$ with $1^{-}\left(4^{++}\right)$. All these $T \bar{T}$ systems could easily be observed if they do exist since the charged state is easy to identify experimentally. Moreover, their total angular momentum $J$ always takes the largest value for these systems. The reason is that the sign for the potential in the isovector case is opposite to that in the isoscalar case. A smaller matrix element for the operator $\mathcal{E}_{1}$ in the diagonal Swave channel leads to a stronger attraction. From the matrix elements in Table 7, the smallest value usually corresponds to the largest $J$. Compared to the isoscalar states, the values of the cutoff $\Lambda$ in the isovector case are all around $4-5 \mathrm{GeV}$. From the experience of the deuteron $(\Lambda \sim 1 \mathrm{GeV})$, this large cutoff means that the attraction is not so strong. It is not difficult to understand this observation from the potentials, where an isospin factor $\mathcal{G}(I)$ always exists. The magnitude of the factor (and thus the potential) in the isovector case is smaller than that in the isoscalar case by a factor of three. As a result, one usually finds more binding solutions in the isoscalar case. Further studies of the possibility of the existence for the isovector states are required. 
When we ignore the channel couplings among $D_{1} \bar{D}_{1}$, $D_{1} \bar{D}_{2}^{*}$, and $D_{2}^{*} \bar{D}_{2}^{*}$, the above results indicate that the $D_{1} \bar{D}_{1}$ with $I^{G}\left(J^{P C}\right)=0^{+}\left(0^{++}\right), D_{1} \bar{D}_{2}^{*}$ with $0^{-}\left(1^{+-}\right), 0^{+}\left(1^{++}\right)$, and $D_{2}^{*} \bar{D}_{2}^{*}$ with $0^{+}\left(0^{++}\right), 0^{-}\left(1^{+-}\right)$can be filtrated out as the molecule candidates if the reasonable cutoff in the OPE model is around $1 \mathrm{GeV}$.

Searching for these prime molecular candidates is very interesting in the future. In the following we also discuss their decay behavior. We present all the allowed S-wave two-body decay modes. The decay channels of the $D_{1} \bar{D}_{1}$ molecular state with $0^{+}\left(0^{++}\right)$include $D \bar{D}, D^{*} \bar{D}^{*}, \eta_{c}(n S) \eta(n=1,2)$, and $\psi(n S) \omega(n=1,2,3)$. For the $D_{1} \bar{D}_{2}^{*}$ state with $0^{-}\left(1^{+-}\right)$, its decay modes are $D \bar{D}^{*}, D^{*} \bar{D}^{*}, \psi(n S) \eta(n=1,2,3,4)$, and $\eta_{c}(n S) \omega(n=1,2)$, while the other $D_{1} \bar{D}_{2}^{*}$ state with $0^{+}\left(1^{++}\right)$can decay into $D \bar{D}^{*}$ and $\psi(n S) \omega(n=1,2,3)$. $D \bar{D}, D^{*} \bar{D}^{*}, \eta_{c}(n S) \eta(n=1,2)$, and $\psi(n S) \omega(n=1,2,3)$ would be the main decay channels for the $D_{2}^{*} \bar{D}_{2}^{*}$ state with $0^{+}\left(0^{++}\right)$. Additionally, the $D_{2}^{*} \bar{D}_{2}^{*}$ state with $0^{-}\left(1^{+-}\right)$can decay to $D \bar{D}^{*}, D^{*} \bar{D}^{*}, \psi(n S) \eta(n=1,2,3,4)$, and $\eta_{c}(n S) \omega$ $(n=1,2)$.

\subsection{The coupled system}

Since the charmed meson masses in the $T$ doublet $\left(D_{1}, D_{2}^{*}\right)$ are very close, it is very necessary to consider the coupledchannel effects for various systems. In Table 3, we present the possible channels to be considered in this work. The states with $J^{P C}=1^{++}, 2^{+-}, 3^{++}$, and $4^{++}$appear only in either $D_{1} \bar{D}_{2}^{*}$ or $D_{2}^{*} \bar{D}_{2}^{*}$ system, which have been discussed in the above subsection.

Compared with Eqs. (3)-(5), several $D$-wave channels are omitted in Table 3 since their contributions are small in our calculation. For illustration, we present the contributions from each S-wave and D-wave channel to the pure $D_{1} \bar{D}_{2}^{*}$ and $D_{2}^{*} \bar{D}_{2}^{*}$ systems for some cases In Table 4 . It is obvious that the contributions from the neglected channels, such as $\left|{ }^{5} D_{1}\right\rangle$, $\left|{ }^{7} D_{1}\right\rangle,\left|{ }^{7} D_{2}\right\rangle,\left|{ }^{9} D_{2}\right\rangle$, and $\left|{ }^{5} D_{3}\right\rangle$, are small (less than $0.6 \%$ ) in the $J=1$ case. In the coupled-channel cases, the contributions of the above neglected $D$-wave $D_{1} \bar{D}_{2}^{*}$ and $D_{2}^{*} \bar{D}_{2}^{*}$ channels are even smaller than those for the pure systems. We have confirmed that the omission of these tiny D-wave channels does not affect the main results after solving the coupled Schrödinger equation.

Now, the binding energy is defined relative to the $D_{1} \bar{D}_{1}$ threshold. One has to add $\left(M_{D_{2}^{*}}-M_{D_{1}}\right)$ to the kinetic term in Eq. (18) for the $D_{1} \bar{D}_{2}^{*}$ channels and $2\left(M_{D_{2}^{*}}-M_{D_{1}}\right)$ for the $D_{2}^{*} \bar{D}_{2}^{*}$ channels in solving the Schrödinger equation for the coupled system.

To get the final potentials, one repeats the procedure to obtain Eq. (15) with the relevant transition amplitudes. For the $J^{P C}=0^{++}$case, we have

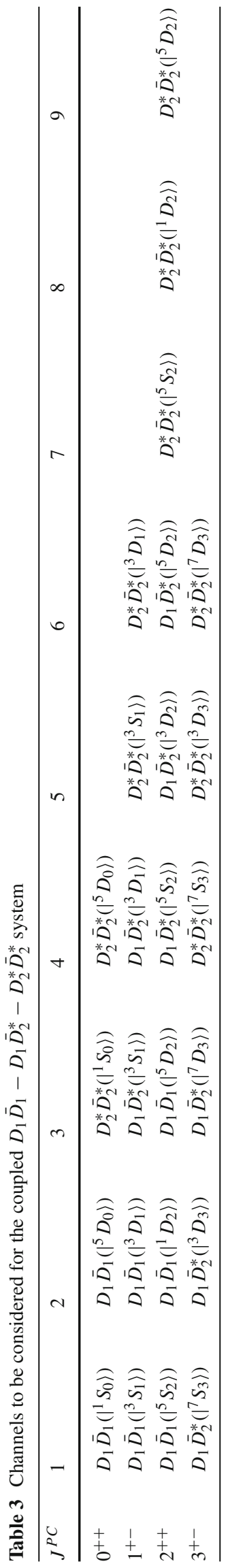


Table 4 The probabilities of each channel for the selected pure $D_{1} \bar{D}_{2}^{*}$ and $D_{2}^{*} \bar{D}_{2}^{*}$ systems

\begin{tabular}{|c|c|c|c|c|c|c|c|c|}
\hline \multirow[t]{2}{*}{$D_{1} \bar{D}_{2}^{*}$} & \multicolumn{8}{|c|}{$\underline{I}^{G}\left(J^{P C}\right)$} \\
\hline & \multicolumn{2}{|c|}{$0^{-}\left(1^{+-}\right)$} & \multicolumn{2}{|c|}{$0^{+}\left(2^{++}\right)$} & \multicolumn{2}{|c|}{$0^{-}\left(3^{+-}\right)$} & \multicolumn{2}{|c|}{$1^{+}\left(3^{+-}\right)$} \\
\hline$\Lambda(\mathrm{GeV})$ & & 1.42 & & 2.40 & & 3.13 & & 4.81 \\
\hline S-wave contribution $(\%)$ & $\left|{ }^{3} S_{1}\right\rangle$ & 99.68 & $\left|{ }^{5} S_{2}\right\rangle$ & 97.38 & $\left|{ }^{7} S_{3}\right\rangle$ & 92.52 & $\left|{ }^{7} S_{3}\right\rangle$ & 98.30 \\
\hline \multirow[t]{3}{*}{ D-wave contribution (\%) } & $\left|{ }^{3} D_{1}\right\rangle$ & 0.05 & $\left.\left.\right|^{3} D_{2}\right\rangle$ & $\sim 0$ & $\left|{ }^{3} D_{3}\right\rangle$ & 0.16 & $\left.\left.\right|^{3} D_{3}\right\rangle$ & 0.51 \\
\hline & $\left|{ }^{5} D_{1}\right\rangle$ & $\sim 0$ & $\left|{ }^{5} D_{2}\right\rangle$ & 2.62 & $\left|{ }^{5} D_{3}\right\rangle$ & $\sim 0$ & $\left|{ }^{5} D_{3}\right\rangle$ & $\sim 0$ \\
\hline & $\left|{ }^{7} D_{1}\right\rangle$ & 0.27 & $\left|{ }^{7} D_{2}\right\rangle$ & $\sim 0$ & $\left|{ }^{7} D_{3}\right\rangle$ & 7.33 & $\left|{ }^{7} D_{3}\right\rangle$ & 1.54 \\
\hline \multirow[t]{2}{*}{$D_{2}^{*} \bar{D}_{2}^{*}$} & \multicolumn{8}{|c|}{$I^{G}\left(J^{P C}\right)$} \\
\hline & \multicolumn{2}{|c|}{$0^{-}\left(1^{+-}\right)$} & \multicolumn{2}{|c|}{$0^{+}\left(2^{++}\right)$} & \multicolumn{2}{|c|}{$0^{-}\left(3^{+-}\right)$} & \multicolumn{2}{|c|}{$1^{+}\left(3^{+-}\right)$} \\
\hline$\Lambda(\mathrm{GeV})$ & & 1.32 & & 1.84 & & 2.90 & & $\ldots$ \\
\hline S-wave contribution (\%) & $\left|{ }^{3} S_{1}\right\rangle$ & 98.53 & $\left|{ }^{5} S_{2}\right\rangle$ & 97.74 & $\left|{ }^{7} S_{3}\right\rangle$ & 94.25 & $\left|{ }^{7} S_{3}\right\rangle$ & $\ldots$ \\
\hline \multirow[t]{3}{*}{ D-wave contribution (\%) } & $\left|{ }^{3} D_{1}\right\rangle$ & 0.90 & $\left|{ }^{1} D_{2}\right\rangle$ & 0.04 & $\left.\left.\right|^{3} D_{3}\right\rangle$ & 0.54 & $\left|{ }^{3} D_{3}\right\rangle$ & $\ldots$ \\
\hline & \multirow[t]{2}{*}{$\left|{ }^{7} D_{1}\right\rangle$} & \multirow[t]{2}{*}{0.58} & $\left|{ }^{5} D_{2}\right\rangle$ & 1.75 & \multirow[t]{2}{*}{$\left|{ }^{7} D_{3}\right\rangle$} & \multirow[t]{2}{*}{5.21} & \multirow[t]{2}{*}{$\left|{ }^{7} D_{3}\right\rangle$} & \multirow[t]{2}{*}{$\ldots$} \\
\hline & & & $\left|{ }^{9} D_{2}\right\rangle$ & 0.48 & & & & \\
\hline
\end{tabular}

The notation $\cdots$ means that no binding solution is found

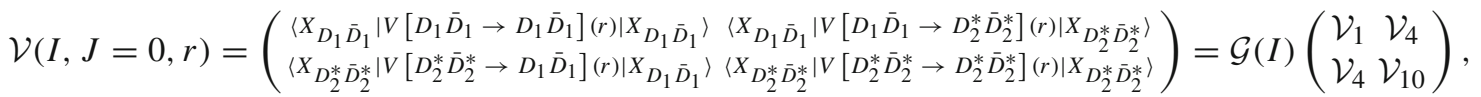

where $\mathcal{V}_{4}$ is a 2 by 2 matrix. The base of the whole $4 \times 4$ matrix is the column matrix composed of the four scalar states in Table 3.

Similarly, the final potentials for the cases $J^{P C}=1^{+-}$, $2^{++}$, and $3^{+-}$are

$$
\begin{aligned}
& \mathcal{V}(I, J=1, r) \\
& \quad=\mathcal{G}(I)\left(\begin{array}{ccc}
\mathcal{V}_{1} & \frac{1}{\sqrt{2}} \mathcal{V}_{2}+\frac{c}{\sqrt{2}} \mathcal{V}_{3} & \mathcal{V}_{4} \\
\frac{1}{\sqrt{2}} \mathcal{V}_{2}+\frac{c}{\sqrt{2}} \mathcal{V}_{3} & \mathcal{V}_{5}+c \mathcal{V}_{6} & \frac{1}{\sqrt{2}} \mathcal{V}_{7}+\frac{c}{\sqrt{2}} \mathcal{V}_{9} \\
\mathcal{V}_{4} & \frac{1}{\sqrt{2}} \mathcal{V}_{7}+\frac{c}{\sqrt{2}} \mathcal{V}_{9} & \mathcal{V}_{10}
\end{array}\right), \\
& \mathcal{V}(I, J=2, r) \\
& \quad=\mathcal{G}(I)\left(\begin{array}{ccc}
\mathcal{V}_{1} & \frac{1}{\sqrt{2}} \mathcal{V}_{2}-\frac{c}{\sqrt{2}} \mathcal{V}_{3} & \mathcal{V}_{4} \\
\frac{1}{\sqrt{2}} \mathcal{V}_{2}-\frac{c}{\sqrt{2}} \mathcal{V}_{3} & \mathcal{V}_{5}-c \mathcal{V}_{6} & \frac{1}{\sqrt{2}} \mathcal{V}_{7}-\frac{c}{\sqrt{2}} \mathcal{V}_{9} \\
\mathcal{V}_{4} & \frac{1}{\sqrt{2}} \mathcal{V}_{7}-\frac{c}{\sqrt{2}} \mathcal{V}_{9} & \mathcal{V}_{10}
\end{array}\right),
\end{aligned}
$$

and

$$
\begin{aligned}
& \mathcal{V}(I, J=3, r) \\
& \quad=\mathcal{G}(I)\left(\begin{array}{cc}
\mathcal{V}_{5}+c \mathcal{V}_{6} & \frac{1}{\sqrt{2}} \mathcal{V}_{7}+\frac{c}{\sqrt{2}} \mathcal{V}_{9} \\
\frac{1}{\sqrt{2}} \mathcal{V}_{7}+\frac{c}{\sqrt{2}} \mathcal{V}_{9} & \mathcal{V}_{10}
\end{array}\right),
\end{aligned}
$$

respectively. The bases of these potential matrices (also the order of elements) are completely determined by the channels in Table 3. Since we ignore small contributions from several channels, the dimension of $\mathcal{V}_{5,6,10}$ here is smaller than that in Eqs. (13) and (14). Note that we do not give $\mathcal{V}_{8}$ and $\mathcal{V}_{11}$ explicitly as in Eq. (13), since they are equal to $\mathcal{V}_{5}$ and $\mathcal{V}_{6}$, respectively. One may find the expressions of these 11 potentials $\mathcal{V}_{k}(k=1,2, \ldots, 11)$ in Appendix A.
Following the same procedure to solve the bound-state problem, one gets the possible binding energies and RMS radius. In Table 5, we present the numerical results for the coupled $T \bar{T}$ systems. The probabilities $\left(p_{i}(\%)\right)$ for each channel are also given. There are six bound-state solutions with the quantum numbers $I^{G}\left(J^{P C}\right)=0^{+}\left(0^{++}\right), 0^{-}\left(1^{+-}\right)$, $0^{+}\left(2^{++}\right), 1^{-}\left(2^{++}\right), 0^{-}\left(3^{+-}\right)$, and $1^{+}\left(3^{+-}\right)$. In Fig. 2, we illustrate the cutoff dependence for the binding energy $E$ and the RMS radius $r_{\mathrm{RMS}}$.

The coupled system with $J=0$ includes four channels $D_{1} \bar{D}_{1}\left(\left|{ }^{1} S_{0}\right\rangle\right), D_{1} \bar{D}_{1}\left(\left|{ }^{5} D_{0}\right\rangle\right), D_{2}^{*} \bar{D}_{2}^{*}\left(\left|{ }^{1} S_{0}\right\rangle\right)$, and $D_{2}^{*} \bar{D}_{2}^{*}\left(\left.\right|^{5}\right.$ $\left.\left.D_{0}\right\rangle\right)$. It is a good molecular candidate since the cutoff parameter $\Lambda$ is around $1 \mathrm{GeV}$ and consistent with the experience from the deuteron. The mass of the candidate is expected to be below the $D_{1} \bar{D}_{1}$ threshold (around $4840 \mathrm{MeV}$ ). Moreover, it is a typical $S$-wave bound state as the channel $D_{1} \bar{D}_{1}\left(\left|{ }^{1} S_{0}\right\rangle\right)$ dominates exclusively and the $D_{2}^{*} \bar{D}_{2}^{*}$ channels have small contributions around several percents.

Six channels are considered for the state with $0^{-}\left(1^{+-}\right)$: $\left.\left.\left.D_{1} \bar{D}_{1}\left(\left.\right|^{3} S_{1}\right\rangle\right), D_{1} \bar{D}_{1}\left(\left.\right|^{3} D_{1}\right\rangle\right), D_{1} \bar{D}_{2}^{*}\left(\left|{ }^{3} S_{1}\right\rangle\right), D_{1} \bar{D}_{2}^{*}\left(\left.\right|^{3} D_{1}\right\rangle\right)$, $\left.D_{2}^{*} \bar{D}_{2}^{*}\left(\left.\right|^{3} S_{1}\right\rangle\right)$, and $\left.D_{2}^{*} \bar{D}_{2}^{*}\left(\left.\right|^{3} D_{1}\right\rangle\right)$. There are small components of $D_{1} \bar{D}_{2}^{*}$ since the probabilities of $D_{1} \bar{D}_{2}^{*}\left(\left|{ }^{3} S_{1}\right\rangle\right)\left(p_{3}\right)$ and $\left.D_{1} \bar{D}_{2}^{*}\left(\left.\right|^{3} D_{1}\right\rangle\right)\left(p_{4}\right)$ are both around zero even with a larger binding energy about $10 \mathrm{MeV}$. For the remaining four channels, the contributions from the S-wave $D_{1} \bar{D}_{1}\left(\left|{ }^{3} S_{1}\right\rangle\right)$ and $\left.D_{2}^{*} \bar{D}_{2}^{*}\left(\left.\right|^{3} S_{1}\right\rangle\right)$ play a leading role in this state. With the binding energy becoming larger and larger, the $D_{2}^{*} \bar{D}_{2}^{*}$ chan- 
Table 5 The binding solutions (binding energy $E$ and root-mean-square radius $r_{\mathrm{RMS}}$ ) after the coupled-channel effects are considered

\begin{tabular}{|c|c|c|c|c|c|c|c|c|c|c|c|c|}
\hline$I^{G}\left(J^{P C}\right)$ & $\Lambda$ & $E$ & $r_{\mathrm{RMS}}$ & $p_{1}(\%)$ & $p_{2}(\%)$ & $p_{3}(\%)$ & $p_{4}(\%)$ & $p_{5}(\%)$ & $p_{6}(\%)$ & $p_{7}(\%)$ & $p_{8}(\%)$ & $p_{9}(\%)$ \\
\hline & & & & $\left.\left.D_{1} \bar{D}_{1}\right|^{1} S_{0}\right\rangle$ & $\left.\left.D_{1} \bar{D}_{1}\right|^{5} D_{0}\right\rangle$ & $\left.\left.D_{2}^{*} \bar{D}_{2}^{*}\right|^{1} S_{0}\right\rangle$ & $\left.\left.D_{2}^{*} \bar{D}_{2}^{*}\right|^{5} D_{0}\right\rangle$ & & & & & \\
\hline \multirow[t]{2}{*}{$0^{+}\left(0^{++}\right)$} & 1.30 & -3.84 & 1.59 & 97.91 & 0.82 & 1.26 & 0.01 & & & & & \\
\hline & 1.40 & -11.46 & 0.98 & 95.46 & 0.90 & 3.60 & 0.03 & & & & & \\
\hline \multirow[t]{2}{*}{$1^{-}\left(0^{++}\right)$} & $\ldots$ & $\ldots$ & $\ldots$ & $\ldots$ & $\ldots$ & $\ldots$ & $\ldots$ & & & & & \\
\hline & & & & $\left.\left.D_{1} \bar{D}_{1}\right|^{3} S_{1}\right\rangle$ & $\left.\left.D_{1} \bar{D}_{1}\right|^{3} D_{1}\right\rangle$ & $\left.\left.D_{1} \bar{D}_{2}^{*}\right|^{3} S_{1}\right\rangle$ & $\left.\left.D_{1} \bar{D}_{2}^{*}\right|^{3} D_{1}\right\rangle$ & $\left.\left.D_{2}^{*} \bar{D}_{2}^{*}\right|^{3} S_{1}\right\rangle$ & $\left.\left.D_{2}^{*} \bar{D}_{2}^{*}\right|^{3} D_{1}\right\rangle$ & & & \\
\hline \multirow[t]{2}{*}{$0^{-}\left(1^{+-}\right)$} & 1.78 & -3.13 & 1.73 & 82.66 & 1.44 & $\sim 0$ & $\sim 0$ & 15.70 & 0.20 & & & \\
\hline & 1.84 & -10.69 & 0.98 & 60.04 & 1.26 & $\sim 0$ & $\sim 0$ & 38.16 & 0.54 & & & \\
\hline \multirow[t]{2}{*}{$1^{+}\left(1^{+-}\right)$} & $\ldots$ & $\ldots$ & $\ldots$ & $\ldots$ & $\ldots$ & $\ldots$ & $\ldots$ & $\ldots$ & $\ldots$ & & & \\
\hline & & & & $D_{1} \bar{D}_{1}\left|{ }^{5} S_{2}\right\rangle$ & $\left.\left.D_{1} \bar{D}_{1}\right|^{1} D_{2}\right\rangle$ & $\left.\left.D_{1} \bar{D}_{1}\right|^{5} D_{2}\right\rangle$ & $\left.\left.D_{1} \bar{D}_{2}^{*}\right|^{5} S_{2}\right\rangle$ & $\left.\left.D_{1} \bar{D}_{2}^{*}\right|^{3} D_{2}\right\rangle$ & $\left.\left.D_{1} \bar{D}_{2}^{*}\right|^{5} D_{2}\right\rangle$ & $D_{2}^{*} \bar{D}_{2}^{*}\left|{ }^{5} S_{2}\right\rangle$ & $\left.\left.D_{2}^{*} \bar{D}_{2}^{*}\right|^{1} D_{2}\right\rangle$ & $\left.\left.D_{2}^{*} \bar{D}_{2}^{*}\right|^{5} D_{2}\right\rangle$ \\
\hline \multirow[t]{2}{*}{$0^{+}\left(2^{++}\right)$} & 1.97 & -2.27 & 1.24 & 30.46 & 0.08 & 1.00 & 34.53 & $\sim 0$ & 0.13 & 33.19 & 0.27 & 0.34 \\
\hline & 1.98 & -4.13 & 0.86 & 22.28 & 0.07 & 0.87 & 38.04 & $\sim 0$ & 0.14 & 37.91 & 0.31 & 0.39 \\
\hline \multirow[t]{3}{*}{$1^{-}\left(2^{++}\right)$} & 3.96 & -2.45 & 1.83 & 94.91 & 0.20 & 1.12 & 3.11 & 0.10 & $\sim 0$ & 0.49 & $\sim 0$ & $\sim 0$ \\
\hline & 4.12 & -9.46 & 0.95 & 90.78 & 0.31 & 1.70 & 5.92 & 0.17 & $\sim 0$ & 1.00 & $\sim 0$ & $\sim 0$ \\
\hline & & & & $\left.\left.D_{1} \bar{D}_{2}^{*}\right|^{7} S_{3}\right\rangle$ & $\left.\left.D_{1} \bar{D}_{2}^{*}\right|^{3} D_{3}\right\rangle$ & $\left.\left.D_{1} \bar{D}_{2}^{*}\right|^{7} D_{3}\right\rangle$ & $\left.\left.D_{2}^{*} \bar{D}_{2}^{*}\right|^{7} S_{3}\right\rangle$ & $\left.\left.D_{2}^{*} \bar{D}_{2}^{*}\right|^{3} D_{3}\right\rangle$ & $\left.\left.D_{2}^{*} \bar{D}_{2}^{*}\right|^{7} D_{3}\right\rangle$ & & & \\
\hline \multirow[t]{2}{*}{$0^{-}\left(3^{+-}\right)$} & 3.16 & -3.31 & 1.86 & 91.90 & 0.16 & 7.94 & $\sim 0$ & $\sim 0$ & $\sim 0$ & & & \\
\hline & 3.37 & -9.89 & 1.17 & 87.97 & 0.21 & 11.82 & $\sim 0$ & $\sim 0$ & $\sim 0$ & & & \\
\hline \multirow[t]{2}{*}{$1^{+}\left(3^{+-}\right)$} & 4.79 & -2.08 & 2.02 & 98.43 & 0.14 & 1.43 & $\sim 0$ & $\sim 0$ & $\sim 0$ & & & \\
\hline & 4.98 & -7.85 & 1.07 & 97.48 & 0.23 & 2.33 & $\sim 0$ & $\sim 0$ & $\sim 0$ & & & \\
\hline
\end{tabular}

$p_{i}(\%)$ denotes the probability for the $i$ th channel. $E, r_{\mathrm{RMS}}$, and $\Lambda$ are in units of $\mathrm{MeV}$, fm, and $\mathrm{GeV}$, respectively. The notation $\cdots$ means that no binding solution is found
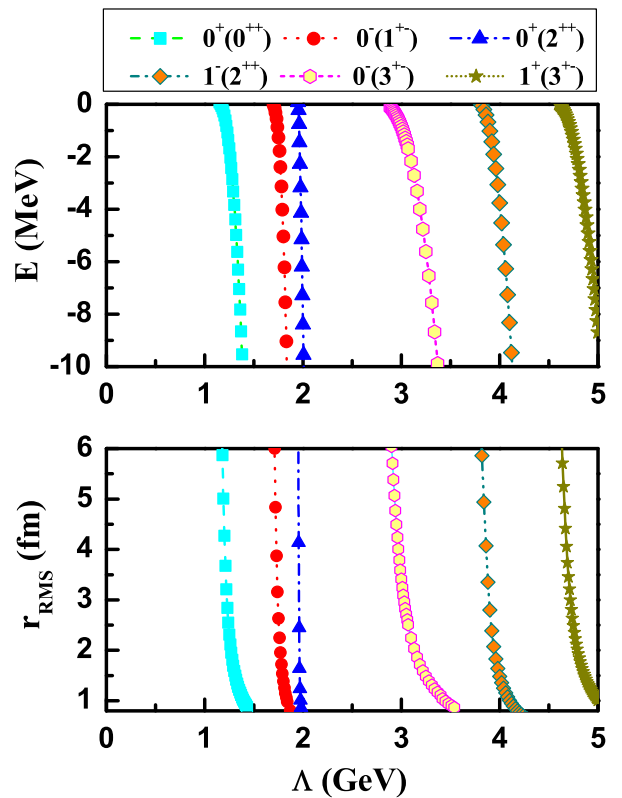

Fig. 2 The $\Lambda$ dependence of the bound-state solutions (the binding energy $E$ and the root-mean-square radius $r_{\mathrm{RMS}}$ ) for the $T \bar{T}$ systems with the coupled-channel effect

nel will play a more significant and increasing role. However, the chance for the $0^{-}\left(1^{+-}\right)$state to be detected by experiments may be lower than that for the $0^{+}\left(0^{++}\right)$as the cutoff $\Lambda\left[0^{-}\left(1^{+-}\right)\right]>\Lambda\left[0^{+}\left(0^{++}\right)\right]$when the binding energies are fixed at the same level.
For the state with $0^{+}\left(2^{++}\right)$, the coupled-channel effects due to $D_{1} \bar{D}_{2}^{*}$ and $D_{2}^{*} \bar{D}_{2}^{*}$ are important since the probabilities for $D_{1} \bar{D}_{1}, D_{1} \bar{D}_{2}^{*}$, and $D_{2}^{*} \bar{D}_{2}^{*}$ are relatively close. The dominant channels are all S-wave $\left|{ }^{5} S_{2}\right\rangle$ with probability around $99 \%$. It can also be a good molecular candidate since the cutoff is around $2 \mathrm{GeV}$.

The above three solutions are all isoscalar states and the masses are all around the $D_{1} \bar{D}_{1}$ threshold. The general feature does not change compared to the previous case but the coupled-channel effects lower the binding energies. For the remaining cases, the solutions with $1^{-}\left(2^{++}\right), 0^{-}\left(3^{+-}\right)$, and $1^{+}\left(3^{+-}\right)$correspond to the typical $D_{1} \bar{D}_{1}, D_{1} \bar{D}_{2}^{*}$, and $D_{1} \bar{D}_{2}^{*}$ bound state, since their dominant channels are $D_{1} \bar{D}_{1}\left(\left|{ }^{5} S_{2}\right\rangle\right)$, $D_{1} \bar{D}_{2}^{*}\left(\left|{ }^{7} S_{3}\right\rangle\right)$ and $\left.D_{1} \bar{D}_{2}^{*}\left({ }^{7} S_{3}\right\rangle\right)$, respectively. However, the corresponding cutoff is far from the usual value around $1 \mathrm{GeV}$.

\section{Summary}

In this work, we have studied the interactions of the $T \bar{T}$-type molecular systems within the framework of the one-pionexchange model. We have found the bound-state solutions for some $T \bar{T}$-type molecular systems. There exist possible $T \bar{T}$-type molecular states, which are summarized in Table 6 for the reader's convenience.

In Table 6, we adopt the same criterion as that in Ref. [35] and show the existence possibility of these bound-state solutions with asterisks. A state with more $\star$ symbols implies 
Table 6 Summary of the $T \bar{T}$ systems

\begin{tabular}{|c|c|c|c|c|c|c|c|}
\hline \multicolumn{3}{|c|}{ Pure system } & \multicolumn{3}{|c|}{ Pure system } & \multicolumn{2}{|c|}{ Coupled system } \\
\hline & $I^{G}\left(J^{P C}\right)$ & Remark & & $I^{G}\left(J^{P C}\right)$ & Remark & $I^{G}\left(J^{P C}\right)$ & Remark \\
\hline \multirow[t]{6}{*}{$D_{1} \bar{D}_{1}$} & $0^{+}\left(0^{++}\right)$ & $\star \star \star \star$ & $D_{2}^{*} \bar{D}_{2}^{*}$ & $0^{+}\left(0^{++}\right)$ & $\star \star \star \star$ & $0^{+}\left(0^{++}\right)$ & $\star \star \star \star$ \\
\hline & $0^{-}\left(1^{+-}\right)$ & $\star \star \star$ & & $0^{-}\left(1^{+-}\right)$ & $\star \star \star \star$ & $0^{-}\left(1^{+-}\right)$ & $\star \star \star$ \\
\hline & $0^{+}\left(2^{++}\right)$ & $\star$ & & $0^{+}\left(2^{++}\right)$ & $\star \star \star$ & $0^{+}\left(2^{++}\right)$ & $\star \star \star$ \\
\hline & $1^{-}\left(2^{++}\right)$ & $\star$ & & $0^{-}\left(3^{+-}\right)$ & $\star \star$ & $1^{-}\left(2^{++}\right)$ & $\star$ \\
\hline & & & & $0^{+}\left(4^{++}\right)$ & $\star$ & $0^{-}\left(3^{+-}\right)$ & $\star \star$ \\
\hline & & & & $1^{-}\left(4^{++}\right)$ & $\star$ & $1^{+}\left(3^{+-}\right)$ & $\star$ \\
\hline \multirow[t]{4}{*}{$D_{1} \bar{D}_{2}^{*}$} & $0^{-}(1)^{+-}$ & $\star \star \star \star$ & $D_{1} \bar{D}_{2}^{*}$ & $0^{+}(1)^{++}$ & $\star \star \star \star$ & & \\
\hline & $0^{-}(2)^{+-}$ & $\star \star$ & & $0^{+}(2)^{++}$ & $\star \star \star$ & & \\
\hline & $0^{-}(3)^{+-}$ & $\star \star$ & & $0^{+}(3)^{++}$ & $\star$ & & \\
\hline & $1^{+}(3)^{+-}$ & $\star$ & & $1^{-}(3)^{++}$ & $\star$ & & \\
\hline
\end{tabular}

higher possibility to find this molecular state. Here, the fourstar, three-star, two-star and one-star notations are applied to mark these states, which have bound-state solutions with cutoff $\Lambda<1.5 \mathrm{GeV}, 1.5<\Lambda<2.5 \mathrm{GeV}, 2.5<\Lambda<3.5 \mathrm{GeV}$ and $3.5<\Lambda<5 \mathrm{GeV}$, respectively. Thus, we suggest experiments to focus on these four-star states, which include the $D_{1} \bar{D}_{1}$ state with $I^{G}\left(J^{P C}\right)=0^{+}\left(0^{++}\right)$, the $D_{1} \bar{D}_{2}^{*}$ states with $0^{ \pm}(1)^{+ \pm}$, the $D_{2}^{*} \bar{D}_{2}^{*}$ state with $0^{+}\left(0^{++}\right)$and the $0^{+}\left(0^{++}\right)$ $D_{1} \bar{D}_{1}-D_{1} \bar{D}_{2}^{*}-D_{2}^{*} \bar{D}_{2}^{*}$ coupled state. In other words, one or several resonant structures around the $D_{1} \bar{D}_{1}$ threshold are highly probable.

In the present model we adopt a phenomenological form factor at each interaction vertex with the adjustable cutoff parameter $\Lambda$. The numerical results are sensitive to its value. Because of a lack of available experimental data, we restrict its reasonable range from the experience with the deuteron. The numerical results are preliminary. In principle, the results should be stable with the variation of the cutoff. Probably the consideration of the other meson exchange forces may reduce the sensitivity once the coupling constants can be appropriately determined. Hopefully one can improve the model and make more reliable predictions in the future.

Searching for the exotic multiquark states continues to be a very interesting issue of hadron physics. The present predictions of the hidden-charm molecular states, which are composed of anticharmed and charmed meson in the $T$ doublet, provide useful information for future experimental exploration of them. The present work is only the starting point for the study of the $T \bar{T}$-type molecular systems, which need to be investigated further by other approaches.

Recent experimental observation of the two hidden-charm pentaquarks $P_{c}$ (4380) and $P_{c}$ (4450) [3] enhances our confidence in the existence of the multiquark states. With experimental progress, especially from $\mathrm{LHCb}$ and forthcoming BelleII, we expect that more candidates of the multiquark states will be announced. This field is full of challenges and opportunities for both theorists and experimentalists.
Acknowledgments This Project is supported by the National Natural Science Foundation of China under Grants Nos. 11222547, 11275115, 11175073 , and 11575008 .

Open Access This article is distributed under the terms of the Creative Commons Attribution 4.0 International License (http://creativecomm ons.org/licenses/by/4.0/), which permits unrestricted use, distribution, and reproduction in any medium, provided you give appropriate credit to the original author(s) and the source, provide a link to the Creative Commons license, and indicate if changes were made.

Funded by $\mathrm{SCOAP}^{3}$.

\section{Appendix A: Relevant subpotentials}

We list the subpotentials $\hat{\mathcal{V}}_{1}-\hat{\mathcal{V}}_{11}$ used in the paper. For convenience, we define

$$
\begin{array}{ll}
\Lambda_{1}=\sqrt{\Lambda^{2}-\left(m_{D_{2}^{*}}-m_{D_{1}}\right)^{2}}, & m_{\pi 1}=\sqrt{m_{\pi}^{2}-\left(m_{D_{2}^{*}}-m_{D_{1}}\right)^{2}}, \\
\Lambda_{2}=\sqrt{\Lambda^{2}-\left(\frac{m_{D_{2}^{*}}^{2}-m_{D_{1}}^{2}}{4 m_{D_{1}}}\right)^{2}}, \quad m_{\pi 2}=\sqrt{m_{\pi}^{2}-\left(\frac{m_{D_{2}^{*}}^{2}-m_{D_{1}}^{2}}{4 m_{D_{1}}}\right)^{2}}, \\
\Lambda_{3}=\sqrt{\Lambda^{2}-\left(\frac{m_{D_{2}^{*}}^{2}-m_{D_{1}}^{2}}{4 m_{D_{2}^{*}}^{*}}\right)^{2}}, \quad m_{\pi 3}=\sqrt{m_{\pi}^{2}-\left(\frac{m_{D_{2}^{*}}^{2}-m_{D_{1}}^{2}}{4 m_{D_{2}^{*}}^{*}}\right)^{2}},
\end{array}
$$

and

$$
\begin{aligned}
Y(\Lambda, m, r) & =\frac{1}{4 \pi r}\left(e^{-m r}-e^{-\Lambda r}\right)-\frac{\Lambda^{2}-m^{2}}{8 \pi \Lambda} e^{-\Lambda r}, \\
S(\hat{r}, \vec{a}, \vec{b}) & =3(\hat{r} \cdot \vec{a})(\hat{r} \cdot \vec{b})-\vec{a} \cdot \vec{b}
\end{aligned}
$$

with $\hat{r}=\vec{r} /|\vec{r}|$.

The first potential is for the $D_{1} \bar{D}_{1}$ system,

$$
\begin{aligned}
\hat{\mathcal{V}}_{1} & \equiv \mathcal{V}\left[D_{1}(1) \bar{D}_{1}(2) \rightarrow D_{1}(3) \bar{D}_{1}(4)\right](r) \\
& =\frac{25}{108} \frac{k^{2}}{f_{\pi}^{2}}\left\{\mathcal{E}_{1} \nabla^{2}+\mathcal{S}_{1} r \frac{\mathrm{d}}{\mathrm{d} r} \frac{1}{r} \frac{\mathrm{d}}{\mathrm{d} r}\right\} Y\left(\Lambda, m_{\pi}, r\right),
\end{aligned}
$$


where the spin-spin operator is defined as $\mathcal{E}_{1}=\left(\vec{\epsilon}_{1} \times \vec{\epsilon}_{3}^{\dagger}\right)$. $\left(\vec{\epsilon}_{2} \times \vec{\epsilon}_{4}^{\dagger}\right)$ and the tensor operator is $\mathcal{S}_{1}=S\left(\hat{r}, \vec{\epsilon}_{1} \times \vec{\epsilon}_{3}^{\dagger}, \vec{\epsilon}_{2} \times \vec{\epsilon}_{4}^{\dagger}\right)$. $\mathcal{V}, \mathcal{E}$, and $\mathcal{S}$ have the same subscript while the subscripts 1 , 2,3 , and 4 of the polarization vectors refer to the incoming meson (1), incoming meson (2), outgoing meson (3), and outgoing meson (4) in the t-channel scattering process, respectively.

The other potentials are

$$
\begin{aligned}
\hat{\mathcal{V}}_{2} & =\mathcal{V}\left[D_{1}(1) \bar{D}_{1}(2) \rightarrow D_{1}(3) \bar{D}_{2}^{*}(4)\right](r) \\
& =-i \frac{5}{18 \sqrt{6}} \frac{k^{2}}{f_{\pi}^{2}}\left\{\mathcal{E}_{2} \nabla^{2}+\mathcal{S}_{2} r \frac{\mathrm{d}}{\mathrm{d} r} \frac{1}{r} \frac{\mathrm{d}}{\mathrm{d} r}\right\} Y\left(\Lambda_{2}, m_{\pi 2}, r\right),
\end{aligned}
$$

with $\mathcal{E}_{2}=\sum_{c, d} C_{1, c ; 1, d}^{2, c+d}\left(\vec{\epsilon}_{2} \cdot \vec{\epsilon}_{4 c}^{\dagger}\right)\left(\vec{\epsilon}_{1} \times \vec{\epsilon}_{3}^{\dagger}\right) \cdot \vec{\epsilon}_{4 d}^{\dagger}$ and $\mathcal{S}_{2}=$ $\sum_{c, d} C_{1, c ; 1, d}^{2, c+d}\left(\vec{\epsilon}_{2} \cdot \vec{\epsilon}_{4 c}^{\dagger}\right) S\left(\hat{r}, \vec{\epsilon}_{1} \times \vec{\epsilon}_{3}^{\dagger}, \vec{\epsilon}_{4 d}^{\dagger}\right)$,

$$
\begin{aligned}
\hat{\mathcal{V}}_{3} & =\mathcal{V}\left[D_{1}(1) \bar{D}_{1}(2) \rightarrow D_{2}^{*}(3) \bar{D}_{1}(4)\right](r) \\
& =i \frac{5}{18 \sqrt{6}} \frac{k^{2}}{f_{\pi}^{2}}\left\{\mathcal{E}_{3} \nabla^{2}+\mathcal{S}_{3} r \frac{\mathrm{d}}{\mathrm{d} r} \frac{1}{r} \frac{\mathrm{d}}{\mathrm{d} r}\right\} Y\left(\Lambda_{2}, m_{\pi 2}, r\right),
\end{aligned}
$$

with $\mathcal{E}_{3}=\sum_{c, d} C_{1, c ; 1, d}^{2, c+d}\left(\vec{\epsilon}_{1} \cdot \vec{\epsilon}_{3 c}^{\dagger}\right)\left(\vec{\epsilon}_{2} \times \vec{\epsilon}_{4}^{\dagger}\right) \cdot \vec{\epsilon}_{3 d}^{\dagger}$ and $\mathcal{S}_{3}=$ $\sum_{c, d} C_{1, c ; 1, d}^{2, c+d}\left(\vec{\epsilon}_{1} \cdot \vec{\epsilon}_{3 c}^{\dagger}\right) S\left(\hat{r}, \vec{\epsilon}_{2} \times \vec{\epsilon}_{4}^{\dagger}, \vec{\epsilon}_{3 d}^{\dagger}\right)$,

$$
\begin{aligned}
\hat{\mathcal{V}}_{4} & =\mathcal{V}\left[D_{1}(1) \bar{D}_{1}(2) \rightarrow D_{2}^{*}(3) \bar{D}_{2}^{*}(4)\right](r) \\
& =\frac{1}{18} \frac{k^{2}}{f_{\pi}^{2}}\left\{\mathcal{E}_{4} \nabla^{2}+\mathcal{S}_{4} r \frac{\mathrm{d}}{\mathrm{d} r} \frac{1}{r} \frac{\mathrm{d}}{\mathrm{d} r}\right\} Y\left(\Lambda, m_{\pi}, r\right),
\end{aligned}
$$

with $\mathcal{E}_{4}=\sum_{c, d, i, j} C_{1, c ; 1, d}^{2, c+d} C_{1, m ; 1, n}^{2, m+n}\left(\vec{\epsilon}_{1} \cdot \vec{\epsilon}_{3 c}^{\dagger}\right)\left(\vec{\epsilon}_{2} \cdot \vec{\epsilon}_{4 m}^{\dagger}\right)\left(\vec{\epsilon}_{3 d}^{\dagger}\right.$. $\left.\vec{\epsilon}_{4 n}^{\dagger}\right) \cdot \vec{\epsilon}_{3 d}^{\dagger}$ and $\mathcal{S}_{4}=\sum_{c, d, i, j} C_{1, c ; 1, d}^{2, c+d} C_{1, m ; 1, n}^{2, m+n}\left(\vec{\epsilon}_{1} \cdot \vec{\epsilon}_{3 c}^{\dagger}\right)\left(\vec{\epsilon}_{2}\right.$. $\left.\vec{\epsilon}_{4 m}^{\dagger}\right) S\left(\hat{r}, \vec{\epsilon}_{3 d}^{\dagger}, \vec{\epsilon}_{4 n}^{\dagger}\right)$,

$$
\begin{aligned}
\hat{\mathcal{V}}_{5} & =\mathcal{V}\left[D_{1}(1) \bar{D}_{2}^{*}(2) \rightarrow D_{1}(1) \bar{D}_{2}^{*}(2)\right](r) \\
& =\frac{5}{18} \frac{k^{2}}{f_{\pi}^{2}}\left\{\mathcal{E}_{5} \nabla^{2}+\mathcal{S}_{5} r \frac{\mathrm{d}}{\mathrm{d} r} \frac{1}{r} \frac{\mathrm{d}}{\mathrm{d} r}\right\} Y\left(\Lambda, m_{\pi}, r\right),
\end{aligned}
$$

with $\mathcal{E}_{5}=\sum_{c, d, m, n} C_{1, c ; 1, d}^{2, c+d} C_{1, m ; 1, n}^{2, m+n}\left(\vec{\epsilon}_{2 c} \cdot \vec{\epsilon}_{4 m}^{\dagger}\right)\left[\left(\vec{\epsilon}_{1} \times \vec{\epsilon}_{3}^{\dagger}\right)\right.$. $\left.\left(\vec{\epsilon}_{2 d} \times \vec{\epsilon}_{4 n}^{\dagger}\right)\right]$ and $\mathcal{S}_{5}=\sum_{c, d, m, n} C_{1, c ; 1, d}^{2, c+d} C_{1, m ; 1, n}^{2, m+n}\left(\vec{\epsilon}_{2 c}\right.$. $\left.\vec{\epsilon}_{4 m}^{\dagger}\right) S\left(\hat{r}, \vec{\epsilon}_{1} \times \vec{\epsilon}_{3}^{\dagger}, \vec{\epsilon}_{2 d} \times \vec{\epsilon}_{4 n}^{\dagger}\right)$,

$$
\begin{aligned}
\hat{\mathcal{V}}_{6} & =\mathcal{V}\left[D_{1}(1) \bar{D}_{2}^{*}(2) \rightarrow D_{2}^{*}(3) \bar{D}_{1}(4)\right](r) \\
& =\frac{1}{18} \frac{k^{2}}{f_{\pi}^{2}}\left\{\mathcal{E}_{6} \nabla^{2}+\mathcal{S}_{6} r \frac{\mathrm{d}}{\mathrm{d} r} \frac{1}{r} \frac{\mathrm{d}}{\mathrm{d} r}\right\} Y\left(\Lambda_{1}, m_{\pi 1}, r\right),
\end{aligned}
$$

with $\mathcal{E}_{6}=\sum_{c, d, m, n} C_{1, c ; 1, d}^{2, c+d} C_{1, m ; 1, n}^{2, m+n}\left(\vec{\epsilon}_{1} \cdot \vec{\epsilon}_{3 c}^{\dagger}\right)\left(\vec{\epsilon}_{2 m} \cdot \vec{\epsilon}_{4}^{\dagger}\right)\left(\vec{\epsilon}_{3 d}^{\dagger}\right.$. $\left.\vec{\epsilon}_{2 n}\right)$ and $\mathcal{S}_{6}=\sum_{c, d, m, n} C_{1, c ; 1, d}^{2, c+d} C_{1, m ; 1, n}^{2, m+n}\left(\vec{\epsilon}_{1} \cdot \vec{\epsilon}_{3 c}^{\dagger}\right)\left(\vec{\epsilon}_{2 m}\right.$. $\left.\vec{\epsilon}_{4}^{\dagger}\right) S\left(\hat{r}, \vec{\epsilon}_{3 d}^{\dagger}, \vec{\epsilon}_{2 n}\right)$,

$$
\begin{aligned}
\hat{\mathcal{V}}_{7} & =\mathcal{V}\left[D_{1}(1) \bar{D}_{2}^{*}(2) \rightarrow D_{2}^{*}(3) \bar{D}_{2}^{*}(4)\right](r) \\
& =\frac{i}{3 \sqrt{6}} \frac{k^{2}}{f_{\pi}^{2}}\left\{\mathcal{E}_{7} \nabla^{2}+\mathcal{S}_{7} r \frac{\mathrm{d}}{\mathrm{d} r} \frac{1}{r} \frac{\mathrm{d}}{\mathrm{d} r}\right\} Y\left(\Lambda_{3}, m_{\pi 3}, r\right),
\end{aligned}
$$

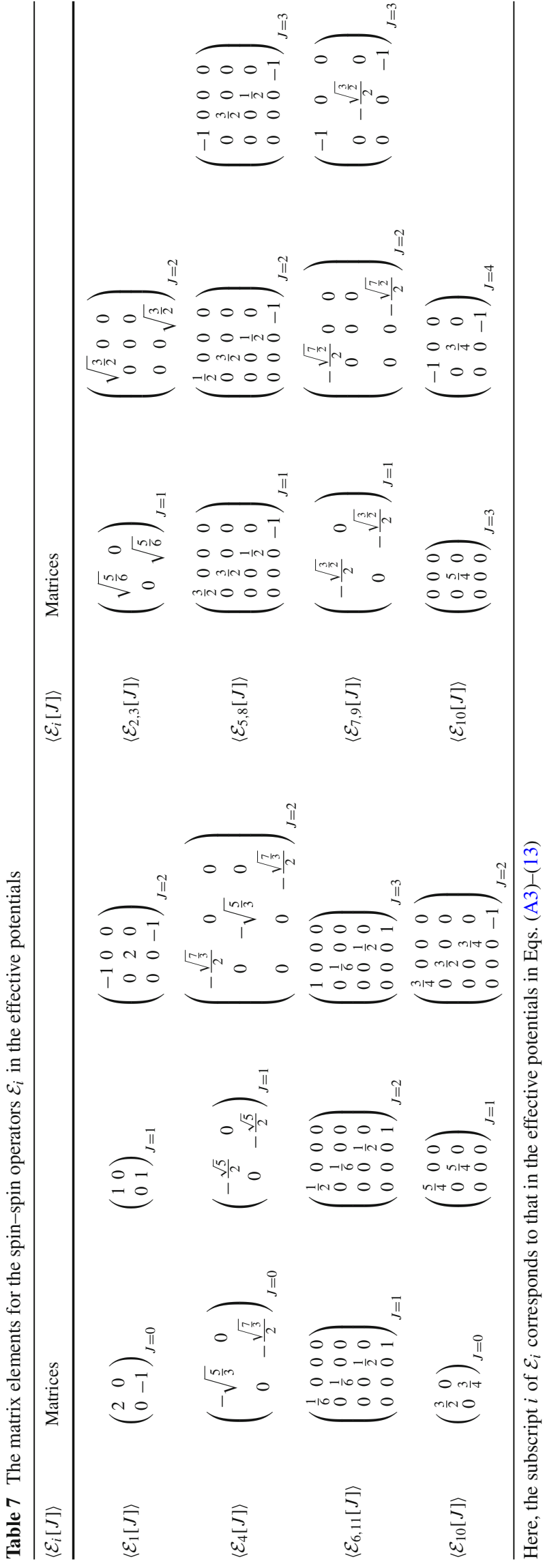


with $\mathcal{E}_{7}=\sum_{c, d, h, l, m, n} C_{1, c ; 1, d}^{2, c+d} C_{1, h ; 1, l}^{2, h+l} C_{1, m ; 1, n}^{2, m+n}\left(\vec{\epsilon}_{1} \cdot \vec{\epsilon}_{3 c}^{\dagger}\right)\left(\vec{\epsilon}_{2 h}\right.$. $\left.\vec{\epsilon}_{4 m}^{\dagger}\right)\left[\vec{\epsilon}_{3 d}^{\dagger} \cdot\left(\vec{\epsilon}_{2 l} \times \vec{\epsilon}_{4 n}^{\dagger}\right)\right]$ and $\mathcal{S}_{7}=\sum_{c, d, m, n} C_{1, c ; 1, d}^{2, c+d} C_{1, m ; 1, n}^{2, m+n}\left(\vec{\epsilon}_{1}\right.$. $\left.\vec{\epsilon}_{3 c}^{\dagger}\right)\left(\vec{\epsilon}_{2 h} \cdot \vec{\epsilon}_{4 m}^{\dagger}\right) S\left(\hat{r}, \vec{\epsilon}_{3 d}^{\dagger}, \vec{\epsilon}_{2 l} \times \vec{\epsilon}_{4 n}^{\dagger}\right)$,

$$
\begin{aligned}
\hat{\mathcal{V}}_{8} & =\mathcal{V}\left[D_{2}^{*}(1) \bar{D}_{1}(2) \rightarrow D_{2}^{*}(3) \bar{D}_{1}(4)\right](r) \\
& =\frac{5}{18} \frac{k^{2}}{f_{\pi}^{2}}\left\{\mathcal{E}_{8} \nabla^{2}+\mathcal{S}_{8} r \frac{\mathrm{d}}{\mathrm{d} r} \frac{1}{r} \frac{\mathrm{d}}{\mathrm{d} r}\right\} Y\left(\Lambda, m_{\pi}, r\right),
\end{aligned}
$$

with $\mathcal{E}_{8}=\sum_{c, d, m, n} C_{1, c ; 1, d}^{2, c+d} C_{1, m ; 1, n}^{2, m+n}\left(\vec{\epsilon}_{1 c} \cdot \vec{\epsilon}_{3 m}^{\dagger}\right)\left[\left(\vec{\epsilon}_{2} \times \vec{\epsilon}_{4}^{\dagger}\right)\right.$. $\left.\left(\vec{\epsilon}_{1 d} \times \vec{\epsilon}_{3 n}^{\dagger}\right)\right]$ and $\mathcal{S}_{8}=\sum_{c, d, m, n} C_{1, c ; 1, d}^{2, c+d} C_{1, m ; 1, n}^{2, m+n}\left(\vec{\epsilon}_{1 c}\right.$. $\left.\vec{\epsilon}_{3 m}^{\dagger}\right) S\left(\hat{r}, \vec{\epsilon}_{2} \times \vec{\epsilon}_{4}^{\dagger}, \vec{\epsilon}_{1 d} \times \vec{\epsilon}_{3 n}^{\dagger}\right)$,

$$
\begin{aligned}
\hat{\mathcal{V}}_{9} & =\mathcal{V}\left[D_{2}^{*}(1) \bar{D}_{1}(2) \rightarrow D_{2}^{*}(3) \bar{D}_{2}^{*}(4)\right](r) \\
& =-i \frac{1}{3 \sqrt{6}} \frac{k^{2}}{f_{\pi}^{2}}\left\{\mathcal{E}_{9} \nabla^{2}+\mathcal{S}_{9} r \frac{\mathrm{d}}{\mathrm{d} r} \frac{1}{r} \frac{\mathrm{d}}{\mathrm{d} r}\right\} Y\left(\Lambda_{3}, m_{\pi 3}, r\right),
\end{aligned}
$$

with $\mathcal{E}_{9}=\sum_{c, d, h, l, m, n} C_{1, c ; 1, d}^{2, c+d} C_{1, h ; 1, l}^{2, h+l} C_{1, m ; 1, n}^{2, m+n}\left(\vec{\epsilon}_{2} \cdot \vec{\epsilon}_{4 c}^{\dagger}\right)\left(\vec{\epsilon}_{1 h}\right.$. $\left.\vec{\epsilon}_{3 m}^{\dagger}\right)\left[\vec{\epsilon}_{4 d}^{\dagger} \cdot\left(\vec{\epsilon}_{1 l} \times \vec{\epsilon}_{3 n}^{\dagger}\right)\right]$ and $\mathcal{S}_{9}=\sum_{c, d, h, l, m, n} C_{1, c ; 1, d}^{2, c+d} C_{1, h ; 1, l}^{2, h+l}$
$C_{1, m ; 1, n}^{2, m+n}\left(\vec{\epsilon}_{2} \cdot \vec{\epsilon}_{4 c}^{\dagger}\right)\left(\vec{\epsilon}_{1 h} \cdot \vec{\epsilon}_{3 m}^{\dagger}\right) S\left(\hat{r}, \vec{\epsilon}_{4 d}^{\dagger}, \vec{\epsilon}_{1 l} \times \vec{\epsilon}_{3 n}^{\dagger}\right)$,

$$
\begin{aligned}
\hat{\mathcal{V}}_{10} & =\mathcal{V}\left[D_{2}^{*}(1) \bar{D}_{2}^{*}(2) \rightarrow D_{2}^{*}(3) \bar{D}_{2}^{*}(4)\right](r) \\
& =\frac{1}{3} \frac{k^{2}}{f_{\pi}^{2}}\left\{\mathcal{E}_{10} \nabla^{2}+\mathcal{S}_{10} r \frac{\partial}{\partial r} \frac{1}{r} \frac{\partial}{\partial r}\right\} Y\left(\Lambda, m_{\pi}, r\right),
\end{aligned}
$$

with $\quad \mathcal{E}_{10}=\sum_{c, d, f, g, h, l, m, n} C_{1, c ; 1, d}^{2, c+d} C_{1, f ; 1, g}^{2, f+g} C_{1, h ; 1, l}^{2, h+l}$ $C_{1, m ; 1, n}^{2, m+n}\left(\vec{\epsilon}_{1 c} \cdot \vec{\epsilon}_{3 f}^{\dagger}\right)\left(\vec{\epsilon}_{2 h} \cdot \vec{\epsilon}_{4 m}^{\dagger}\right)\left[\left(\vec{\epsilon}_{1 d} \times \vec{\epsilon}_{3 g}^{\dagger}\right) \cdot\left(\vec{\epsilon}_{2 l} \times \vec{\epsilon}_{4 n}^{\dagger}\right)\right]$ and $\mathcal{S}_{10}=\sum_{c, d, f, g, h, l, m, n} C_{1, c ; 1, d}^{2, c+d} C_{1, f ; 1, g}^{2, f+g} C_{1, h ; 1, l}^{2, h+l} C_{1, m ; 1, n}^{2, m+n}\left(\vec{\epsilon}_{1 c}\right.$. $\left.\vec{\epsilon}_{3 f}^{\dagger}\right)\left(\vec{\epsilon}_{2 h} \cdot \vec{\epsilon}_{4 m}^{\dagger}\right) S\left(\hat{r}, \vec{\epsilon}_{1 d} \times \vec{\epsilon}_{3 g}^{\dagger}, \vec{\epsilon}_{2 l} \times \vec{\epsilon}_{4 n}^{\dagger}\right)$,

$$
\begin{aligned}
\hat{\mathcal{V}}_{11} & =\mathcal{V}\left[D_{2}^{*}(1) \bar{D}_{1}(2) \rightarrow D_{1}(3) \bar{D}_{2}^{*}(4)\right](r) \\
& =\frac{1}{18} \frac{k^{2}}{f_{\pi}^{2}}\left\{\mathcal{E}_{11} \nabla^{2}+\mathcal{S}_{11} r \frac{\mathrm{d}}{\mathrm{d} r} \frac{1}{r} \frac{\mathrm{d}}{\mathrm{d} r}\right\} Y\left(\Lambda_{1}, m_{\pi 1}, r\right)
\end{aligned}
$$

with $\mathcal{E}_{11}=\sum_{c, d, m, n} C_{1, c ; 1, d}^{2, c+d} C_{1, m ; 1, n}^{2, m+n}\left(\vec{\epsilon}_{2} \cdot \vec{\epsilon}_{4 c}^{\dagger}\right)\left(\vec{\epsilon}_{1 m} \cdot \vec{\epsilon}_{3}^{\dagger}\right)\left(\vec{\epsilon}_{4 d}^{\dagger}\right.$. $\left.\vec{\epsilon}_{1 n}\right)$ and $\mathcal{S}_{11}=\sum_{c, d, m, n} C_{1, c ; 1, d}^{2, c+d} C_{1, m ; 1, n}^{2, m+n}\left(\vec{\epsilon}_{2} \cdot \vec{\epsilon}_{4 c}^{\dagger}\right)\left(\vec{\epsilon}_{1 m}\right.$. $\left.\vec{\epsilon}_{3}^{\dagger}\right) S\left(\hat{r}, \vec{\epsilon}_{4 d}^{\dagger}, \vec{\epsilon}_{1 n}\right)$.

Table 8 The matrix elements for the generalized tensor operators $\mathcal{S}_{i}$ in the effective potentials

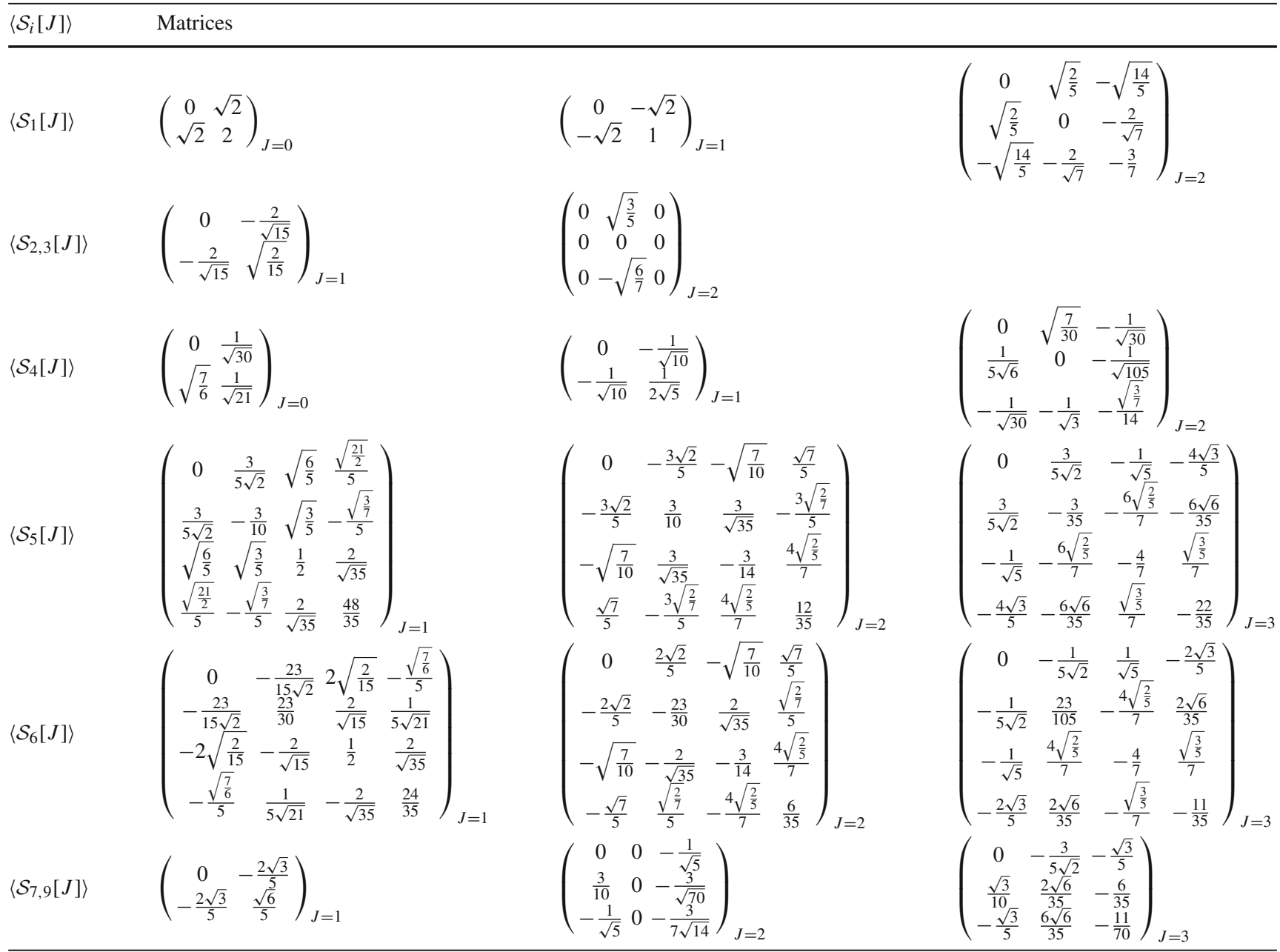


Table 8 continued

$\left\langle\mathcal{S}_{i}[J]\right\rangle \quad$ Matrices

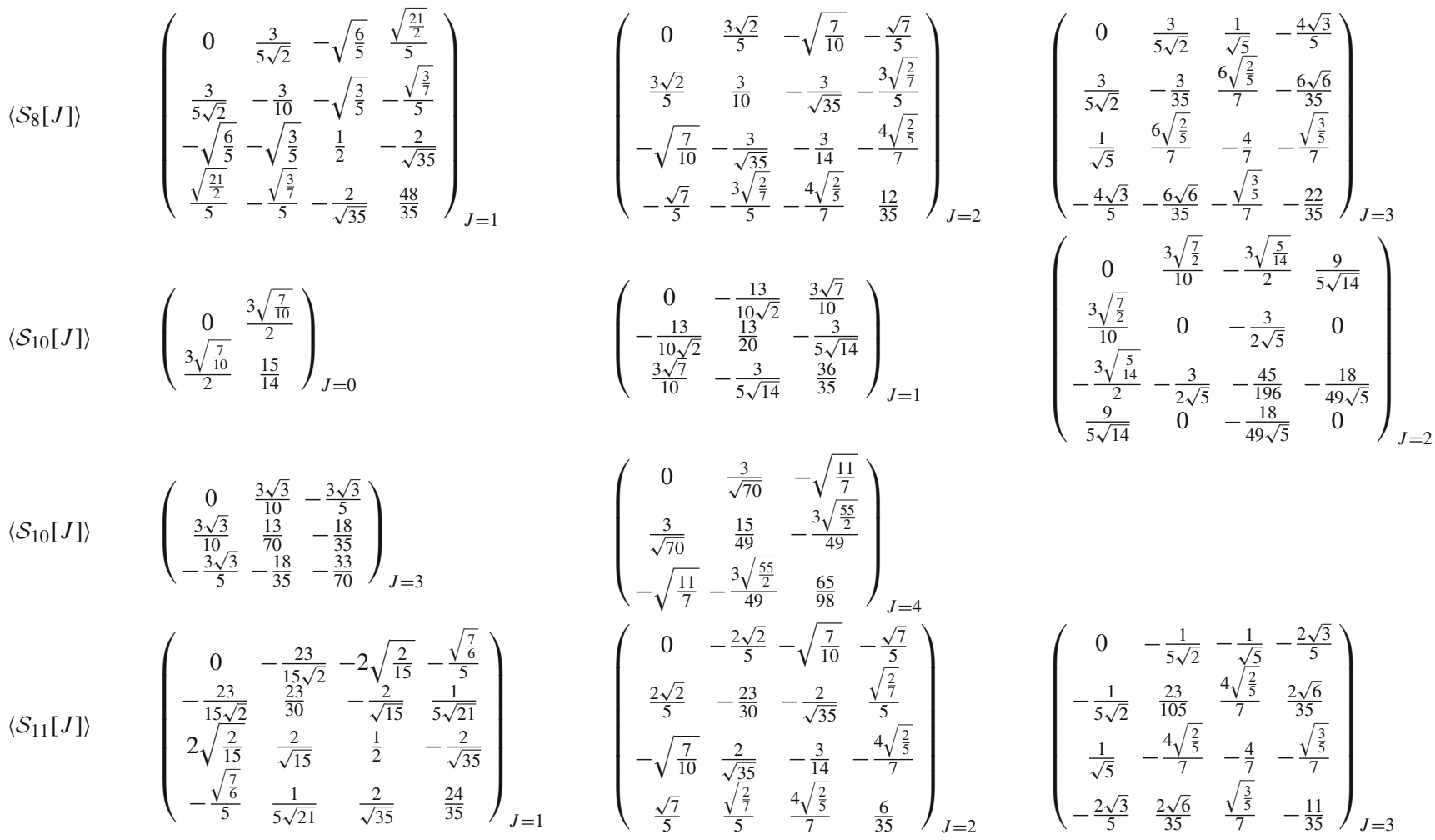

Here, the subscript $i$ of $\mathcal{S}_{i}$ corresponds to that in the effective potentials in Eqs. (A3)-(13)

We present the matrix elements for the spin-spin operators $\mathcal{E}_{i}$ and the generalized tensor operators $\mathcal{S}_{i}$ in Tables 7 and 8 , respectively.

\section{References}

1. M. Ablikim et al. [BESIII Collaboration], Observation of a charged charmoniumlike structure in $e^{+} e^{-} \rightarrow \pi^{+} \pi^{-} J / \psi$ at $\sqrt{s}=4.26$ GeV. Phys. Rev. Lett. 110, 252001 (2013) arXiv:1303.5949 [hepex]

2. Z.Q. Liu et al. [Belle Collaboration], Study of $e^{+} e^{-} \rightarrow \pi^{+} \pi^{-} J / \psi$ and observation of a charged charmoniumlike state at Belle. Phys. Rev. Lett. 110, 252002 (2013) arXiv:1304.0121 [hep-ex]

3. R. Aaij et al. [LHCb Collaboration], Observation of $J / \psi p$ resonances consistent with pentaquark states in $\Lambda_{b}^{0} \rightarrow J / \psi K^{-} p$ Decays, Phys. Rev. Lett. 115, 072001 (2015) arXiv:1507.03414 [hep-ex]

4. R. Chen, X. Liu, X.Q. Li, S.L. Zhu, Identifying exotic hiddencharm pentaquarks. Phys. Rev. Lett. 115, 132002 (2015). arXiv:1507.03704 [hep-ph]

5. H.X. Chen, W. Chen, X. Liu, T.G. Steele, S.L. Zhu, Towards exotic hidden-charm pentaquarks in QCD. Phys. Rev. Lett. 115, 172001 (2015). arXiv:1507.03717 [hep-ph]

6. L. Roca, J. Nieves, E. Oset, The LHCb pentaquark as a $\bar{D}^{*} \Sigma_{c}-$ $\bar{D}^{*} \Sigma_{c}^{*}$ molecular state. arXiv:1507.04249 [hep-ph]

7. A. Mironov, A. Morozov, Is pentaquark doublet a hadronic molecule?. arXiv:1507.04694 [hep-ph]
8. J. He, The $\bar{D} \Sigma_{c}^{*}$ and $\bar{D}^{*} \Sigma_{c}$ interactions and the LHCb hiddencharmed pentaquarks. arXiv:1507.05200 [hep-ph]

9. Y.R. Liu, X. Liu, W.Z. Deng, S.L. Zhu, Is X (3872) really a molecular state? Eur. Phys. J. C 56, 63 (2008). arXiv:0801.3540 [hep-ph]

10. E.S. Swanson, Short range structure in the X(3872). Phys. Lett. B 588, 189 (2004). arXiv:hep-ph/0311229

11. N.A. Tornqvist, Isospin breaking of the narrow charmonium state of Belle at 3872-MeV as a Deuson. Phys. Lett. B 590, 209 (2004). arXiv:hep-ph/0402237

12. N.A. Tornqvist, On Deusons or Deuteron-like Meson Meson bound states. Nuovo Cim. A 107, 2471 (1994). arXiv:hep-ph/9310225

13. N.A. Tornqvist, From the Deuteron to Deusons, an analysis of Deuteron-like Meson Meson bound states. Z. Phys. C 61, 525 (1994). arXiv:hep-ph/9310247

14. N. Li, S.L. Zhu, Isospin breaking, coupled-channel effects and diagnosis of X(3872). Phys. Rev. D 86, 074022 (2012). arXiv:1207.3954 [hep-ph]

15. X. Liu, Z.G. Luo, Y.R. Liu, S.L. Zhu, X(3872) and other possible heavy molecular states. Eur. Phys. J. C 61, 411 (2009). arXiv:0808.0073 [hep-ph]

16. Y.R. Liu, X. Liu, S.L. Zhu, Light pseudoscalar Meson and heavy Meson scattering lengths. Phys. Rev. D 79, 094026 (2009). arXiv:0904.1770 [hep-ph]

17. T. Branz, T. Gutsche, V.E. Lyubovitskij, Hadronic molecule structure of the Y(3940) and Y(4140). Phys. Rev. D 80, 054019 (2009). arXiv:0903.5424 [hep-ph]

18. X. Liu, S.L. Zhu, Y(4143) is probably a molecular partner of Y(3930). Phys. Rev. D 80, 017502 (2009)

19. X. Liu, S.L. Zhu, Phys. Rev. D 85, 019902 (2012). arXiv:0903.2529 [hep-ph] 
20. X. Liu, Z.G. Luo, S.L. Zhu, Novel charmonium-like structures in the $J / \psi \phi$ and $J / \psi \omega$ invariant mass spectra. Phys. Lett. B 699, 341 (2011)

21. X. Liu, Z.G. Luo, S.L. Zhu, Phys. Lett. B 707, 577 (2012). arXiv:1011.1045 [hep-ph]

22. J. He, X. Liu, The open-charm radiative and pionic decays of molecular charmonium Y(4274). Eur. Phys. J. C 72, 1986 (2012). arXiv:1102.1127 [hep-ph]

23. Z.F. Sun, J. He, X. Liu, Z.G. Luo, S.L. Zhu, $Z_{b}(10610)^{ \pm}$and $Z_{b}(10650)^{ \pm}$as the $B^{*} \bar{B}$ and $B^{*} \bar{B}^{*}$ molecular states. Phys. Rev. D 84, 054002 (2011). arXiv:1106.2968 [hep-ph]

24. Z.F. Sun, Z.G. Luo, J. He, X. Liu, S.L. Zhu, A note on the $B^{*} \bar{B}$, $B^{*} \bar{B}^{*}, D^{*} \bar{D}, D^{*} \bar{D}^{*}$, molecular states. Chin. Phys. C 36, 194 (2012)

25. X. Liu, Y.R. Liu, W.Z. Deng, S.L. Zhu, Is $Z^{+}(4430)$ a loosely bound molecular state? Phys. Rev. D 77, 034003 (2008). arXiv:0711.0494 [hep-ph]

26. X. Liu, Y.R. Liu, W.Z. Deng, S.L. Zhu, $Z^{+}(4430)$ as a $D_{1}^{\prime} D^{*}$ $\left(D_{1} D^{*}\right)$ molecular state. Phys. Rev. D 77, 094015 (2008). arXiv:0803.1295 [hep-ph]

27. X.Q. Li, X. Liu, A possible global group structure for exotic states. Eur. Phys. J. C 74, 3198 (2014). arXiv:1409.3332 [hep-ph]

28. M. Karliner, J.L. Rosner, New exotic Meson and Baryon resonances from doubly-heavy hadronic molecules. Phys. Rev. Lett. 115, 122001 (2015). arXiv:1506.06386 [hep-ph]

29. A.V. Manohar, M.B. Wise, Heavy quark physics. Camb. Monogr. Part. Phys. Nucl. Phys. Cosmol. 10, 1 (2000)

30. B. Hu, X.L. Chen, Z.G. Luo, P.Z. Huang, S.L. Zhu, P.F. Yu, $X$. Liu, Possible heavy molecular states composed of a pair of excited charm-strange mesons. Chin. Phys. C 35, 113 (2011). arXiv: 1004.4032 [hep-ph]

31. L.L. Shen, X.L. Chen, Z.G. Luo, P.Z. Huang, S.L. Zhu, P.F. $\mathrm{Yu}, \mathrm{X}$. Liu, The molecular systems composed of the charmed mesons in the $H \bar{S}+$ h.c. doublet. Eur. Phys. J. C 70, 183 (2010). arXiv:1005.0994 [hep-ph]

32. A.A. Filin, A. Romanov, V. Baru, C. Hanhart, Y.S. Kalashnikova, A.E. Kudryavtsev, U.-G. Meissner, A.V. Nefediev, Comment on 'Possibility of Deeply Bound Hadronic Molecules from Single Pion Exchange'. Phys. Rev. Lett. 105, 019101 (2010). arXiv:1004.4789 [hep-ph]

33. F. Close, C. Downum, On the possibility of deeply bound hadronic molecules from single pion exchange. Phys. Rev. Lett. 102, 242003 (2009). arXiv:0905.2687 [hep-ph]

34. Z.C. Yang, Z.F. Sun, J. He, X. Liu, S.L. Zhu, The possible hiddencharm molecular baryons composed of anti-charmed meson and charmed baryon. Chin. Phys. C 36, 6 (2012). arXiv:1105.2901 [hep-ph]

35. Z.F. Sun, X. Liu, M. Nielsen, S.L. Zhu, Hadronic molecules with both open charm and bottom. Phys. Rev. D 85, 094008 (2012). arXiv:1203.1090 [hep-ph]
36. N. Li, Z.F. Sun, X. Liu, S.L. Zhu, Coupled-channel analysis of the possible $D^{(*)} D^{(*)}, \bar{B}^{(*)} \bar{B}^{(*)}$ and $D^{(*)} \bar{B}^{(*)}$ molecular states. Phys. Rev. D 88, 114008 (2013). arXiv: 1211.5007 [hep-ph]

37. R. Chen, Z.F. Sun, X. Liu, S.M. Gerasyuta, Predicting exotic molecular states composed of nucleon and P-wave charmed meson. Phys. Rev. D 90, 034011 (2014). arXiv:1406.7481 [hep-ph]

38. L. Zhao, P.N. Shen, Y.J. Zhang, B.S. Zou, A possible $N N^{*}(1440)$ quasi-molecular state. Eur. Phys. J. A 49, 59 (2013). arXiv: 1302.4790 [hep-ph]

39. K.A. Olive et al., [Particle Data Group Collaboration], Review of particle physics. Chin. Phys. C 38, 090001 (2014)

40. W. Zhu, Y.R. Liu, T. Yao, Is $J^{P C}=3^{-+}$molecule possible? Chin. Phys. C 39, 023101 (2015). arXiv:1302.4496 [hep-ph]

41. Y.R. Liu, Heavy quark spin selection rules in meson-antimeson states. Phys. Rev. D 88, 074008 (2013). arXiv:1304.7467 [hep-ph]

42. H.Y. Cheng, K.C. Yang, Charmless hadronic B decays into a tensor meson. Phys. Rev. D 83, 034001 (2011). arXiv:1010.3309 [hep-ph]

43. G.-J. Ding, Are $Y(4260)$ and $Z_{2}^{+}(4250)$ are $D_{1} D$ or $D_{0} D^{*}$ hadronic molecules? Phys. Rev. D 79, 014001 (2009). arXiv:0809.4818 [hep-ph]

44. C. Isola, M. Ladisa, G. Nardulli, P. Santorelli, Charming penguins in $B \rightarrow K^{*} \pi, K(\rho, \omega, \phi)$ decays. Phys. Rev. D 68, 114001 (2003). arXiv:hep-ph/0307367

45. A.F. Falk, M.E. Luke, Strong decays of excited heavy mesons in chiral perturbation theory. Phys. Lett. B 292, 119 (1992). arXiv:hep-ph/9206241

46. A.G. Abrashkevich, D.G. Abrashkevich, M.S. Kaschiev, I.V. Puzynin, FESSDE, a program for the finite-element solution of the coupled-channel Schrödinger equation using high-order accuracy approximations. Comput. Phys. Commun. 85, 65 (1995)

47. A.G. Abrashkevich, D.G. Abrashkevich, M.S. Kaschiev, I.V. Puzynin, FESSDE 2.2: a new version of a program for the finiteelement solution of the coupled-channel Schrödinger equation using high-order accuracy approximations. Comput. Phys. Commun. 115, 90 (1998)

48. Y.J. Zhang, H.C. Chiang, P.N. Shen, B.S. Zou, Phys. Rev. D 74, 014013 (2006). arXiv:hep-ph/0604271

49. Y.D. Chen, C.F. Qiao, P.N. Shen, Z.Q. Zeng, Revisiting the spectrum of baryonium in heavy baryon chiral perturbation theory. Phys. Rev. D 88, 114007 (2013). arXiv:1305.4713 [hep-ph]

50. C.E. Thomas, F.E. Close, Is $X(3872)$ a molecule? Phys. Rev. D 78, 034007 (2008). arXiv:0805.3653 [hep-ph]

51. I.W. Lee, A. Faessler, T. Gutsche, V.E. Lyubovitskij, $X(3872)$ as a molecular $D D^{*}$ state in a potential model". Phys. Rev. D 80, 094005 (2009). arXiv:0910.1009 [hep-ph] 